\title{
Determination of Interrogating Frequencies to Maximize Electromagnetic Backscatter from Objects with Material Coatings
}

\author{
H.T. Banks, K. Ito, and J. Toivanen* \\ Center for Research in Scientific Computation, Box 8205 \\ North Carolina State University \\ Raleigh, NC 27695-8205.
}

August 27, 2005

\begin{abstract}
The electromagnetic backscattering of a crosscut of a cruise missile coated by a thin homogeneous layer made of radar absorbent material is modeled using a finite element method. Based on the radar cross section and a reflection coefficient, optimization problems are formulated for evaders and interrogators leading to optimal material parameters for the coating and optimal monostatic radar operating frequencies, respectively. Optimal coating materials are constructed for several radar frequencies. Tuning only dielectric permittivity gives a narrow frequency range of high absorption while also tuning magnetic permeability widens it significantly. However the coating layers considered cannot provide substantial reduction of backscattering in the entire frequency range from 0.2 to $1.6 \mathrm{GHz}$. The computational experiments also demonstrate that the reflection coefficient based on a planar geometry can predict well the strength of radar cross section in the sector of interest.
\end{abstract}

Key words: radar cross section, radar absorbent material, optimization, finite element model

\section{Introduction}

In this paper we consider the determination of interrogating frequencies to maximize electromagnetic backscatter from objects with radar absorbent material (RAM) coatings. Particularly we are interested in radar cross sections (RCS) of missile like objects using monostatic radars. This is an important class of problems, since the backscattering from missiles is rather faint compared to ones from airplanes. Thus, a RAM coating on a missile might make it very difficult to detect and the proper choice of interrogation frequency can be crucial for effective interrogation. We present a general mathematical formulation for optimizing the frequencies for detection. It is derived under the assumption that the evader also minimizes the backscattering by choosing optimal permittivity and permeability for the coating material. The formulation can be simplified substantially when the material parameters for the coating are known.

\footnotetext{
*Email: htbanks@ncsu.edu, kito@ncsu.edu, jatoivan@ncsu.edu.
} 
The scientific literature has considered earlier the shape and material optimization for the evader. For example, the papers [9, 12, 22, 23] consider multidisciplinary shape optimization problems for an airfoil where the aim is to minimize the electromagnetic backscattering together with aerodynamical constraints and maximization. In $[11,17]$ an optimization for the shape and material parameters for the coating layer is performed to minimize the RCS in a sector when the shape of outer boundary of the airfoil is fixed. The paper [8] considers the optimization of the material parameters of a coating layer.

In this paper we choose our model geometry to be a crosscut of a cruise missile [13] which is coated with a thin homogeneous layer of radar absorbent material. We construct a finite element model for the transverse magnetic polarization in the frequency range from $0.2 \mathrm{GHz}$ to $1.6 \mathrm{GHz}$. We use linear finite elements on triangulation which is locally fitted to the interfaces and refined in the coating layer in order to provide sufficient accuracy. Furthermore, the computational domain is truncated to be a rectangular domain and a second-order absorbing boundary condition $[1,18]$ is imposed on the artificial boundary. A more detailed description of the finite element method and the solution of the resulting large linear systems is given in [19]. One of our interests is to study the use of the reflection coefficient based on a plane wave analysis to predict the strength of the backscattering in the considered frequency range.

The outline of the paper is as follows. We begin by defining the geometry of the model cruise missile in Section 2. This section contains also the description of the finite element model and the formula for the reflection coefficient. In Section 3, we consider optimization problems for evaders and interrogators. These are discussed in the context of two player zero sum minmax games in Section 4. We report the results of numerical experiments in Section 5. Our conclusions and some suggestions for further investigations are given in the last section.

\section{Model problem}

We study the electromagnetic scattering by a perfectly conducting cruise missile coated by a radar absorbent material (RAM) layer of constant thickness. In this investigation we compute the scattering of a crosscut of a missile in two-dimensions. The interrogating electromagnetic incident wave is assumed to be a transverse magnetic. We simplify the geometry by neglecting fins. We remark that this simplification generally reduces the backscattering and, thus, if we can detect a missile without fins then we can certainly detect the same missile with fins. We model the exhaust nozzle as a cavity with perfectly conducting walls. The dimensions and approximate geometry of the missile are taken from [13]. The length of the missile is 6.25 meters and its diameter is 0.52 meters. The leading edge is modeled as a half sphere. The exhaust cavity is 0.5 meters long and its diameter is 0.255 meters. The geometry is shown in Figure 1.

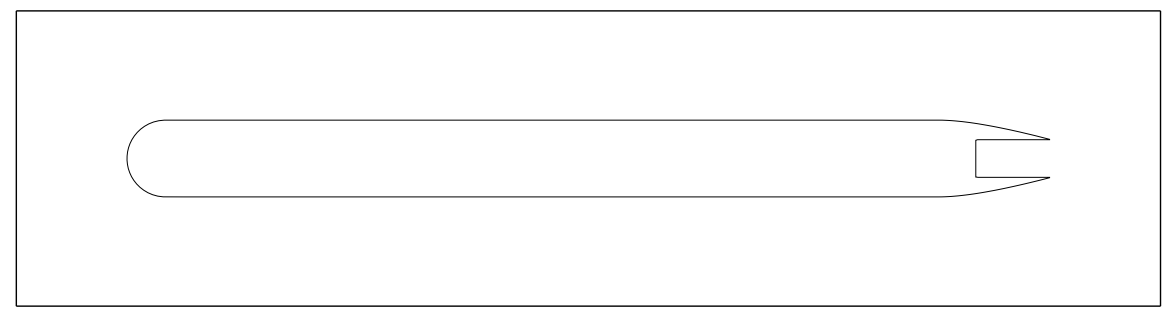

Figure 1: The crosscut of the cruise missile and the computational domain. 
The missile is illuminated by a monostatic radar operating in the frequency range from 0.2 $\mathrm{GHz}$ to $1.6 \mathrm{GHz}$. This means that the wavelength varies in the range from 0.1875 meters to 1.5 meters. Furthermore, the missile is from 4.16 to 33.33 wavelengths long. The interrogating plane wave is such that the angle between the propagation direction of the wave and the centerline of the missile is from $0^{\circ}$ to $30^{\circ}$.

The thickness of a homogeneous RAM layer is chosen to be 2.5 millimeters. This thickness is denoted by $d$ in the following. The relative material permittivity $\epsilon_{r}$ and permeability $\mu_{r}$ are constants in the coating.

\section{$2.1 \quad$ Finite element model}

Let the crosscut plane be the $x_{1} x_{2}$-plane. Due to the transverse magnetic polarization of the electromagnetic field the electric field has the form $(0,0, E)^{T}$ where the function $E$ is constant in the $x_{3}$-direction. In this case Maxwell's equation for a time-harmonic electric field $E$ reduces to a Helmholtz equation. Let us denote the missile by $\Omega$ and its coating layer by $\Omega_{1}$. For a finite element model the exterior domain is truncated to be the rectangle $\Pi=[-1.0,6.75] \times$ $[-1.0,1.0]$ where the leading edge of the missile is at $(-0.25,0.0)$. Here the units are meters. We approximate the Silver-Müller radiation condition by a second-order absorbing boundary condition [1] on $\partial \Pi$; see also $[18,20]$. We split the field $E$ into an incident field $E^{(i)}$ and a scattered field $E^{(s)}$. Thus, we have $E=E^{(i)}+E^{(s)}$. Then the scattered field $E^{(s)}$ satisfies the Helmholtz equation

$$
\begin{aligned}
\nabla \cdot\left(\frac{1}{\mu} \nabla E^{(s)}\right)+\epsilon \omega^{2} E^{(s)} & =-\nabla \cdot\left(\frac{1}{\mu} \nabla E^{(i)}\right)-\epsilon \omega^{2} E^{(i)} & & \text { in } \Pi \backslash \bar{\Omega} \\
E^{(s)} & =-E^{(i)} & & \text { on } \partial \Omega \\
{\left[\frac{1}{\mu} \frac{\partial E}{\partial n}\right]=[E] } & =0 & & \text { on } \partial \Omega_{1} \backslash \partial \Omega \\
\frac{\partial E^{(s)}}{\partial n}-i k E^{(s)}-\frac{i}{2 k} \frac{\partial^{2} E^{(s)}}{\partial s^{2}} & =0 & & \text { on } \partial \Pi \\
\frac{\partial E^{(s)}}{\partial s}-i k \frac{3}{2} E^{(s)} & =0 & & \text { at } C,
\end{aligned}
$$

where $n$ and $s$ denote the normal and tangential directions of the boundary $\partial \Pi$, respectively, and $C$ is the set of the corner points of $\Pi$. Furthermore, $[\cdot]$ denotes the jump. The incident field in air is given by

$$
E^{(i)}=e^{i k\left(x_{1} \cos \alpha+x_{2} \sin \alpha\right)},
$$

where $\alpha$ is the incident angle and $k=2 \pi f / c=\omega / c$ is the interrogation wave number corresponding to the interrogating frequency $f$.

A standard variational formulation for (1) is given in [8]. We perform a finite element discretization using linear finite elements. The mesh step size in air is one centimeter and in the coating layer it is one 16th of a centimeter. The refinement of the mesh is carried out in a conforming manner. Magnified views of the mesh near the leading edge and the upper trailing edge are shown in Figure 2. The mesh consists of 276761 nodes and 529212 triangles. We use a preconditioned iterative GMRES method [19] based a domain decomposition method for solving 
the resulting large systems of linear equations. Our approximation for the far field pattern $F$ is described in [18].
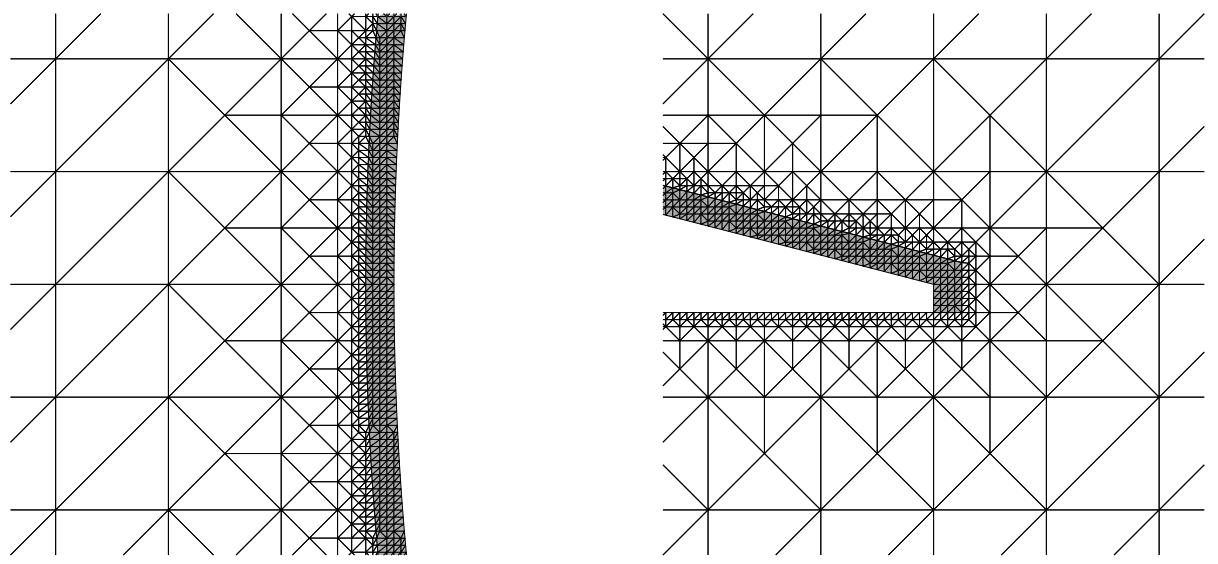

Figure 2: Magnified views of the mesh in the rectangles $[-0.28,-0.24] \times[-0.02,0.02]$ and $[5.98,6.02] \times[0.11,0.15]$. The coating layer is painted with gray.

\subsection{Reflection coefficient model}

The finite element model gives a good but computationally expensive way to study backscattering. A computationally more affordable model is obtained by computing the reflection coefficient for a coated half plane. For this let us consider a perfectly conducting half plane which has a coating layer of thickness $d$ with the relative permittivity $\epsilon_{r}$ and the relative permeability $\mu_{r}$. Let a normally incident electromagnetic wave with the frequency $f$ impinge the half plane. Then the corresponding wavelength $\lambda$ in air is $\lambda=c / f$, where the speed of light is $c=0.3 \times 10^{9}$. The reflection coefficient $R$ for the wave (see $[8,21]$ ) is given by

$$
R=\frac{a+b}{1+a b}
$$

where

$$
a=\frac{\epsilon_{r}-\sqrt{\epsilon_{r} \mu_{r}}}{\epsilon_{r}+\sqrt{\epsilon_{r} \mu_{r}}} \quad \text { and } \quad b=e^{4 i \pi \sqrt{\epsilon_{r} \mu_{r}} f d / c} .
$$

\section{Optimization problems for interrogators and evaders}

\subsection{The strength of backscatter}

We denote the strength of electromagnetic backscattering at the interrogating frequency $f$ with the coating material parameters $\epsilon_{r}$ and $\mu_{r}$ by $B\left(\epsilon_{r}, \mu_{r}, f\right)$. We describe several possible choices for $B$ below. We denote a generic measure over frequencies for the strength of backscattering by $J\left(\epsilon_{r}, \mu_{r}, P, B\right)$ for a coated target with the material parameters $\epsilon_{r}$ and $\mu_{r}$. One natural choice for $J$ is

$$
J_{2}\left(\epsilon_{r}, \mu_{r}, P, B\right)=\int_{I}\left(B\left(\epsilon_{r}, \mu_{r}, f\right)\right)^{2} d P(f),
$$


where $I$ is a set of frequencies and $P$ is a probability measure on $I$. When $P$ is the constant $(1 /$ meas $I)$ this reduces to

$$
J_{2}\left(\epsilon_{r}, \mu_{r}, P, B\right)=\frac{1}{\operatorname{meas} I} \int_{I}\left(B\left(\epsilon_{r}, \mu_{r}, f\right)\right)^{2} d f .
$$

Another measure $J$ which gives the maximum strength of backscattering is

$$
J_{\infty}\left(\epsilon_{r}, \mu_{r}, P, B\right)=\max _{f \in \operatorname{supp} P} B\left(\epsilon_{r}, \mu_{r}, f\right),
$$

where supp $P$ denotes the support of $P$.

The far field pattern of the scattered field (see $[8,14]$ ) describes the strength of backscattering. We denote the far field pattern of the scattered field to the direction $\alpha+180^{\circ}$ by $F_{\alpha}\left(\alpha+180^{\circ} ; f\right)$ when the incident angle is $\alpha$. With a monostatic radar this is the far field pattern of interest. It can be defined (see [8]) as

$$
F_{\alpha}\left(\alpha+180^{\circ} ; f\right)=\lim _{r \rightarrow \infty}\left(\sqrt{8 \pi k r} e^{-i(k r+\pi / 4)} E^{(s)}\left(r \cos \left(\alpha+180^{\circ}\right), r \sin \left(\alpha+180^{\circ}\right) ; f\right)\right),
$$

where $k=2 \pi f / c$ is the interrogation wave number and $E^{(s)}$ is the scattered electromagnetic field. The usual way to illustrate the amplitude of the far field pattern is to plot the radar cross section defined by

$$
\operatorname{RCS}(\alpha ; f)=10 \log _{10}\left(\frac{1}{8 \pi}\left|F_{\alpha}\left(\alpha+180^{\circ} ; f\right)\right|^{2}\right) .
$$

Now one possible definition for the measure of the strength of backscattering at the frequency $f$ is

$$
B_{F}\left(\epsilon_{r}, \mu_{r}, f\right)=\int_{A}\left|F_{\alpha}\left(\alpha+180^{\circ} ; f\right)\right|^{2} d Q(\alpha)
$$

where $A$ is a set of incidence angles and $Q$ is a probability measure on $A$.

An alternative, computationally much cheaper measure of the strength of backscattering is

$$
B_{R}\left(\epsilon_{r}, \mu_{r}, f\right)=\left|R\left(\epsilon_{r}, \mu_{r}, f\right)\right|,
$$

where $R$ is the reflection coefficient defined in (2).

In the discussions and computations reported on below, we will test the use of and compare findings for each of the measures $B_{F}$ of $(8)$ and $B_{R}$ of (9). As we shall see, calculations with $B_{R}$ are much faster than those with $B_{F}$ and are just as accurate for predictions unless the discretization level in the finite element calculations for $E^{(s)}$ are rather fine.

\subsection{Determining optimal frequencies for interrogation and an optimal coating material for evading}

The goal of the interrogator is to find a probability measure $P$ on $I$ in (4) or (6) which maximizes the strength $J$ of the electromagnetic backscattering. There are technological limits on the possible interrogation frequencies and, therefore, the probability measures $P$ should belong to a set of feasible probability measures on $I$ which we denote by $\mathcal{P}$. For example, often a discrete set of frequencies is used for radar interrogation. Thus, in this case $P$ can be a combination 
of Dirac delta measures $\delta_{f_{j}}$ for given frequencies $\left\{f_{j}\right\}$. In any event, $P$ is to be found as the solution of the optimization problem

$$
\max _{P \in \mathcal{P}} J\left(\epsilon_{r}, \mu_{r}, P, B\right) .
$$

A difficulty with the optimization problem (10) is that the material parameters $\epsilon_{r}$ and $\mu_{r}$ are not usually known. We know that the pair $\left(\epsilon_{r}, \mu_{r}\right)$ must belong to a set of feasible material parameters denoted by $\mathcal{M}$. In particular, all $\left(\epsilon_{r}, \mu_{r}\right) \in \mathcal{M}$ should satisfy $\operatorname{Re} \epsilon_{r} \geq 1, \operatorname{Im} \epsilon_{r} \geq 0$, Re $\mu_{r} \geq 1$, and $\operatorname{Im} \mu_{r} \geq 0$. Furthermore, there are also upper limits on the real and imaginary parts of $\epsilon_{r}$ and $\mu_{r}$ which depend on available coating materials.

A smart evader tries to minimize the backscattering while the interrogator tries to maximize it. This leads to the problem

$$
\min _{\left(\epsilon_{r}, \mu_{r}\right) \in \mathcal{M}} \max _{P \in \mathcal{P}} J\left(\epsilon_{r}, \mu_{r}, P, B\right) .
$$

From a computational point of view, the problem (11) is very expensive to solve if $B_{F}$ based on the finite element model is used. It is computationally preferable to use $B_{R}$ based on the reflection coefficient. Therefore, an important question is how well $B_{R}$ predicts the behavior of $B_{F}$.

A more tractable problem for the evader is to assume that the interrogating radar uses some frequency interval which we denote by $I_{D}$. Actually there can be several frequency intervals, but for simplicity we consider here only one interval. Furthermore, it is natural in some cases for the evader to simply assume a uniform probability measure $P$ over $I_{D}$. With (6) measuring the maximum backscattering, this leads to the minimization or design problem for the evader (given the set $I_{D}$ )

$$
\min _{\left(\epsilon_{r}, \mu_{r}\right) \in \mathcal{M}} \max _{f \in I_{D}} B\left(\epsilon_{r}, \mu_{r}, f\right) .
$$

We denote the solution of this problem by $\epsilon_{r}^{*}$ and $\mu_{r}^{*}$.

On the other hand, if the interrogator assumes that the evader has previously performed the minimization (12), then he or she should perform the maximization in $(10)$ at $\left(\epsilon_{r}, \mu_{r}\right)=\left(\epsilon_{r}^{*}, \mu_{r}^{*}\right)$.

\section{Some theoretical considerations}

The problems formulated in the previous section are special cases of classical static zero-sum two player games [10] where the evader controls the $\left(\epsilon_{r}, \mu_{r}\right)$ in $\mathcal{M}$ and the interrogator controls the interrogating frequencies $f \in I$ or $P \in \mathcal{P}(I)$. In such games one defines upper and lower values for the game by

$$
\bar{J}=\inf _{\left(\epsilon_{r}, \mu_{r}\right) \in \mathcal{M}} \sup _{P \in \mathcal{P}} J\left(\epsilon_{r}, \mu_{r}, P, B\right)
$$

and

$$
\underline{J}=\sup _{P \in \mathcal{P}} \inf _{\left(\epsilon_{r}, \mu_{r}\right) \in \mathcal{M}} J\left(\epsilon_{r}, \mu_{r}, P, B\right) .
$$

The first represents a security level for the evader while the latter is a security level for the interrogator. It is readily argued that $\underline{J} \leq \bar{J}$ and if the equality $J^{*}=\underline{J}=\bar{J}$ holds, then $J^{*}$ is called the value of the (static) zero-sum game. Moreover, if there exist $\left(\epsilon_{r}^{*}, \mu_{r}^{*}\right) \in \mathcal{M}$ and $\mathcal{P}^{*} \in \mathcal{P}$ such that

$$
J^{*}=J\left(\epsilon_{r}^{*}, \mu_{r}^{*}, P^{*}\right)=\min _{\left(\epsilon_{r}, \mu_{r}\right) \in \mathcal{M}} J\left(\epsilon_{r}, \mu_{r}, P^{*}\right)=\max _{P \in \mathcal{P}} J\left(\epsilon_{r}^{*}, \mu_{r}^{*}, P\right)
$$


then $\left(\left(\epsilon_{r}^{*}, \mu_{r}^{*}\right), P^{*}\right)$ is a saddle point solution of the game. To investigate theoretical issues in such a context for our problems, one would proceed in a typical fashion by establishing certain regularity and compactness properties. First, one would establish continuity of the mapping on $\mathcal{M} \times I$ defined by $\left(\epsilon_{r}, \mu_{r}, f\right) \rightarrow B\left(\epsilon_{r}, \mu_{r}, f\right)$. For the function $B=B_{R}$ of (9) this continuity is readily obvious. For $B_{F}$ of (8) one first argues continuity for each $\alpha \in A$ of $f \rightarrow F_{\alpha}\left(\alpha+180^{\circ} ; f\right)$ for the far field given by (7). But this reduces to arguing continuity of the solution $E^{(s)}=E^{(s)}(f)$ of the Helmhotz equation (1) which depends on $\alpha$ and $f$ through the incident wave

$$
E^{(i)}(f, \alpha)=\exp \left\{i(2 \pi f / c)\left(x_{1} \cos \alpha+x_{2} \sin \alpha\right)\right\}
$$

and boundary parameters (see (1) and recall $k=2 \pi f / c)$. Since $(f, \alpha) \rightarrow E^{(i)}(f, \alpha)$ is clearly continuous, the arguments reduce to standard continuous dependence arguments for parameters in an elliptic partial differential equation which we do not pursue here.

Once one establishes the required continuity in $f$, one then turns to topologies on $\mathcal{M} \times \mathcal{P}$ and some type of compactness. The obvious topology for $\mathcal{M}$ is the usual Euclidean one, in which typical sets $\mathcal{M}$ (see (13) below) are readily compact. For $\mathcal{P}(I)$ a natural choice is the Prohorov metric topology as used for minimization/inverse problems in $[2,3,7]$. Prohorov metric convergence is weak $k^{*}$ convergence in $\mathcal{P}$ when it is considered as a subset of the topological dual $C_{B}^{*}(I)$ of the space $C_{B}(I)$ of bounded continuous functions on $I$. That is, $P_{k} \rightarrow P$ in the Prohorov metric if and only if

$$
\int_{I} \phi(\nu) d P_{k}(\nu) \rightarrow \int_{I} \phi(\nu) d P(\nu)
$$

for all $\phi \in C_{B}(I)$, i.e., all bounded continuous $\phi$ on $I$. It is known $[2,3]$ that if $I$ is a complete metric space, then $\mathcal{P}$ taken with the Prohorov metric is a complete metric space. Moreover, if $I$ is compact, then so is $\mathcal{P}$. Using these properties and arguments similar to those in $[2,4,5,6,7]$, one can develop well-posedness and approximation results for the minmax problems defined in this paper. We defer pursuit of such a theory to another effort and concentrate here on computational findings

\section{$5 \quad$ Numerical results}

\subsection{Frequencies around $1.0 \mathrm{GHz}$}

We begin by optimizing the material parameters for given frequency intervals $I_{D}$ around 1.0 $\mathrm{GHz}$ which we shall refer to as evader's design frequency intervals. For these minimizations we use each the reflection coefficient based $B_{R}$ and the far field pattern based $B_{F}$, respectively, as $B$ in (12) with a given $I_{D}$. For the finite element based optimization the incident angles in (8) belong to the sector $A=[0,30]^{\circ}$ with the constant weight $1 / 30$ for all directions (i.e., $Q$ is chosen as the uniform distribution on $[0,30])$.

Using a given pair $\left(\epsilon_{r}^{\max }, \mu_{r}^{\max }\right)$ we define the set $\mathcal{M}$ of feasible material parameters in (12) to be

$$
\begin{aligned}
& \mathcal{M}=\left\{\left(\epsilon_{r}, \mu_{r}\right) \mid\right. 1 \leq \operatorname{Re} \epsilon_{r} \leq \operatorname{Re} \epsilon_{r}^{\max }, 0 \leq \operatorname{Im} \epsilon_{r} \leq \operatorname{Im} \epsilon_{r}^{\max }, \\
&\left.1 \leq \operatorname{Re} \mu_{r} \leq \operatorname{Re} \mu_{r}^{\max }, 0 \leq \operatorname{Im} \mu_{r} \leq \operatorname{Im} \mu_{r}^{\max }\right\}
\end{aligned} .
$$

In Table 1 we report the results of these optimizations. In the optimizations of the materials denoted by $1 \mathrm{R}, 2 \mathrm{R}, 1 \mathrm{~F}$, and $2 \mathrm{~F}$ the design frequency interval $I_{D}$ is just the single frequency 
1.0 GHz, i.e., $I_{D}=\{1\}$, while for the material $3 \mathrm{R}$ and $3 \mathrm{~F}$ the interval is $I_{D}=[0.8,1.25]$ GHz. For the materials $1 R$ and $1 F$, only the relative permittivity $\epsilon_{r}$ is optimized, since the relative permeability $\mu_{r}$ is fixed at $1+0 i$. For all optimizations given in Table 1 , we have used $\epsilon_{r}^{\max }=2000+400 i$. In order to simplify notation, we denote $\max _{f \in I_{D}} B_{R}\left(\epsilon_{r}^{*}, \mu_{r}^{*}, f\right)$ and $\max _{f \in I_{D}} B_{F}\left(\epsilon_{r}^{*}, \mu_{r}^{*}, f\right)$ by $\max B_{R}^{D}$ and $\max B_{F}^{D}$, respectively, in the table.

The optimizations based on the reflection coefficient are performed by computing the values of $\tilde{B}_{R}\left(\epsilon_{r}, \mu_{r}\right)=\max _{f \in I_{D}} B_{R}\left(\epsilon_{r}, \mu_{r}, f\right)$ on a grid over the feasible material parameters $\mathcal{M}$ and then choosing the point with the smallest value. For the optimizations of $B_{F}$ based on the finite element model we use a quasi-Newton method with the BFGS update for the approximation of the Hessian [15] with forward finite difference approximations for gradients. As the initial guesses we used the corresponding optimal material parameters computed using $B_{R}$. On the boundaries of the feasible domain $\mathcal{M}$ we projected the search direction to be feasible. The integral in (8) is approximated using the trapezoidal quadrature rule with one degree steps. We compute the maximum of $B$ over $I_{D}$ by taking the maximum of the value of $B$ at the end points of $I_{D}$. For the coating layers and frequency ranges considered here, this gives the correct value for maximizing over all of $I_{D}$.

One evaluation of $B_{F}$ requires about 10 minutes on a PC with Intel Xeon $3.4 \mathrm{GHz} \mathrm{CPU}$ and one optimization run takes a few days depending on the convergence of the quasi-Newton method. The use of the finite element model in the optimization is several orders of magnitude more time consuming than the use of the reflection coefficient $R$. For example, the optimization for the material $1 \mathrm{R}$ based on $R$ using the grid step size 0.1 for $\mathcal{M}$ requires about one minute.

\begin{tabular}{c|c|c|c|c|c|c} 
material & $I_{D}$ & $\mu_{r}^{\max }$ & $\epsilon_{r}^{*}$ & $\mu_{r}^{*}$ & $\max B_{R}^{D}$ & $\max B_{F}^{D}$ \\
\hline \hline no & $\{1\}$ & & $1+0 i$ & $1+0 i$ & $1.00 \times 10^{-0}$ & $6.83 \times 10^{+1}$ \\
\hline \hline 1R & $\{1\}$ & $1+0 i$ & $900.4+38.2 i$ & $1+0 i$ & $1.03 \times 10^{-4}$ & $1.94 \times 10^{-0}$ \\
\hline $2 \mathrm{R}$ & $\{1\}$ & $10+2 i$ & $90.7+21.4 i$ & $10+2 i$ & $4.26 \times 10^{-5}$ & $1.01 \times 10^{-2}$ \\
\hline $3 \mathrm{R}$ & {$[0.8,1.25]$} & $10+2 i$ & $91.64+37.08 i$ & $10+2 i$ & $4.02 \times 10^{-1}$ & $1.35 \times 10^{+1}$ \\
\hline \hline $1 \mathrm{~F}$ & $\{1\}$ & $1+0 i$ & $887.7+39.5 i$ & $1+0 i$ & $1.62 \times 10^{-1}$ & $3.87 \times 10^{-2}$ \\
\hline $2 \mathrm{~F}$ & $\{1\}$ & $10+2 i$ & $90.89+22.0 i$ & $10+2 i$ & $7.84 \times 10^{-3}$ & $5.78 \times 10^{-3}$ \\
\hline $3 \mathrm{~F}$ & {$[0.8,1.25]$} & $10+2 i$ & $85.93+36.12 i$ & $10+2 i$ & $4.38 \times 10^{-1}$ & $1.05 \times 10^{+1}$
\end{tabular}

Table 1: Optimized coating materials $1 \mathrm{R}, 2 \mathrm{R}, 3 \mathrm{R}$ using the reflection coefficient $R$ and $1 \mathrm{~F}, 2 \mathrm{~F}$, $3 \mathrm{~F}$ using the far field pattern $F$ compared to the no coating case.

Once we completed the calculations reported on in Table 1, we used the optimal values $\left(\epsilon_{r}^{*}, \mu_{r}^{*}\right)$ to consider $B_{R}^{*}(f)=B_{R}\left(\epsilon_{r}^{*}, \mu_{r}^{*}, f\right)$ as the interrogating frequency changes over the interval $[.2,1.6]$. In Figure 3 we plot the modulus of this reflection coefficient $B_{R}^{*}(f)$ versus the frequency for the optimized materials $1 \mathrm{R}, 2 \mathrm{R}$, and $3 \mathrm{R}$ in Table 1. Similarly, Figure 5 depicts $B_{F}^{*}(f)=$ $B_{F}\left(\epsilon_{r}^{*}, \mu_{r}^{*}, f\right)$ of $(8)$ for the same materials. In order to make the comparison easier we have also plotted the ratio between $B_{F}^{*}$ for the coated missiles and $B_{F}(1,1, f)$ for the non coated missile in Figure 4. We remark that in both figures the strength of the backscatter is plotted on a $\log$ scale so that the reduction of $R$ from 1 to 0.1 represents a 10 decibel reduction, reduction to 0.01 represents a 20 decibel reduction, etc. The behavior the curves are similar in Figures 3 and 4 . The $B_{R}^{*}$ based reflection coefficient predicts stronger backscattering than does that based on the far field pattern $B_{F}^{*}$. Based on Figure 4 for the material $1 \mathrm{R}$ the reduction in the 
backscattering is about 10 decibels for interrogating frequencies within $\pm 2 \%$ of $1.0 \mathrm{GHz}$. For the material $2 \mathrm{R}$ the reduction is about 10 and 20 decibels for interrogating frequencies within $\pm 15 \%$ and $\pm 5 \%$, respectively, of $1.0 \mathrm{GHz}$. Thus, the ability to optimize also the permeability $\mu_{r}$ widens significantly the frequency range of effective reduction of backscattering.

If the evader has only optimized the relative permittivity $\epsilon_{r}$ for $I_{D}=\{1.0\} \mathrm{GHz}$ then the interrogator only needs to use frequencies, say, more than $10 \%$ off from $1.0 \mathrm{GHz}$ to obtain strong backscattering from the missile. The interrogator must vary the radar frequency from $1.0 \mathrm{GHz}$ significantly more when the evader has tuned both permittivity and permeability of the coating material. Thus, a dual frequency radar using, for example, interrogating frequencies 0.4 and 1.4 $\mathrm{GHz}$ should be able to detect missiles with any of the optimized coating layers for $I_{D}=\{1.0\}$ GHz.

In order to see more clearly the behavior of $B_{F}^{*}$ near the minima the curves for the materials $1 \mathrm{R}$ and $2 \mathrm{R}$ are shown in Figure 6 with an enlarged scale. We have also plotted $B_{F}^{*}$ for the materials $1 \mathrm{~F}$ and $2 \mathrm{~F}$ in this figure. The minimum for the material $1 \mathrm{R}$ is reached about $0.7 \%$ below $1.0 \mathrm{GHz}$. A question arises is this due to the finite element discretization error or for some reason. To answer this we refined the finite element mesh by halving mesh step size and then we again computed the values of $B_{F}^{*}$ for the material $1 \mathrm{R}$. The resulting curve is also shown in Figure 6. For this refined computation the minimum is about $0.2 \%$ below $1.0 \mathrm{GHz}$. This convergence with refinement toward $1.0 \mathrm{GHz}$ suggests that the perceived difference is mainly due to the finite element discretization error. Hence, for these particular backscattering problems the plane wave analysis gives a very accurate prediction of the frequency range leading to the minimal backscattering.

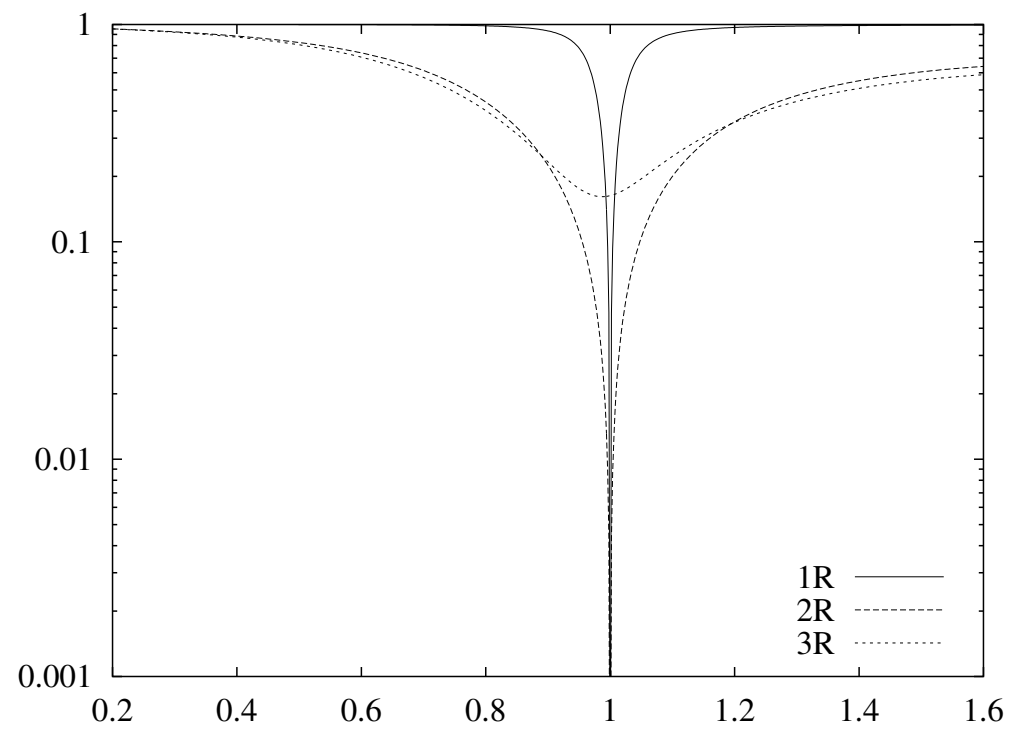

Figure 3: The strength of backscatter $B_{R}^{*}$ as a function of the interrogating frequency $f$ based on the reflection coefficient $R$ for different coating layers. 


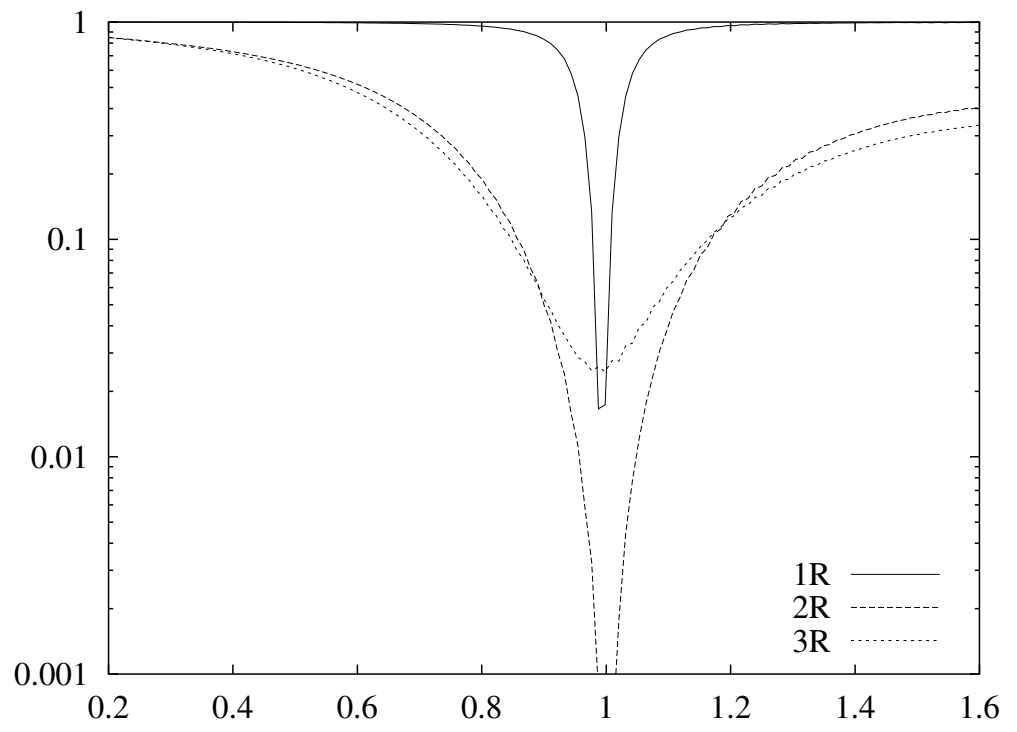

Figure 4: The relative strength of backscatter $B_{F}\left(\epsilon_{r}^{*}, \mu_{r}^{*}, f\right) / B_{F}(1,1, f)$ as a function of the interrogating frequency $f$ for different coating layers.

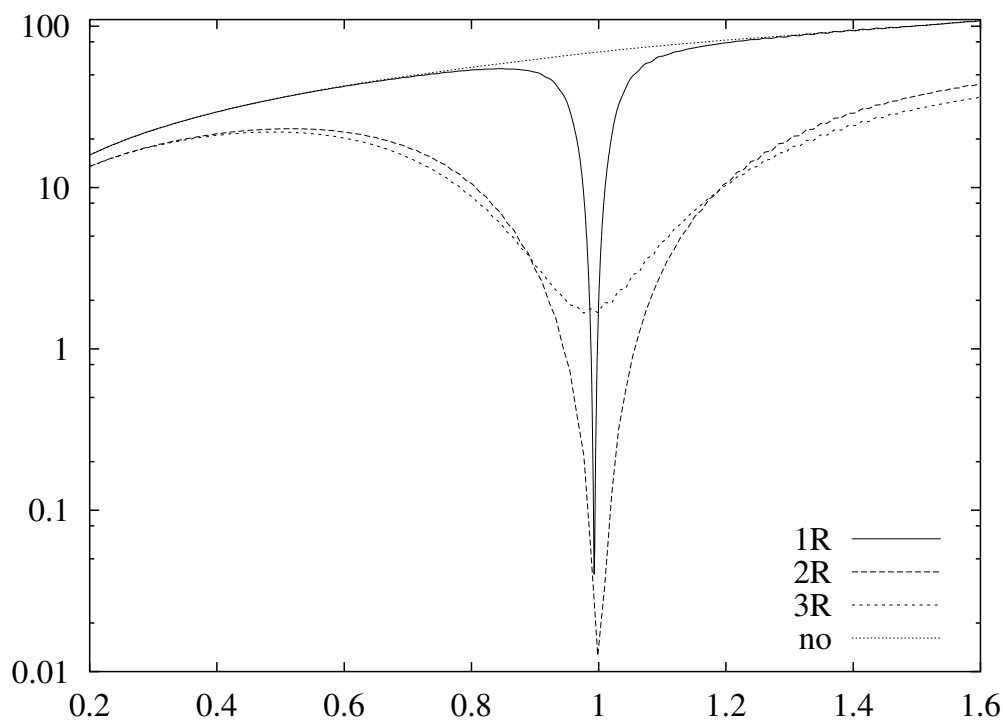

Figure 5: The strength of backscatter $B_{F}^{*}$ as a function of the interrogating frequency $f$ based on the far field pattern $F$ for different coating layers. 


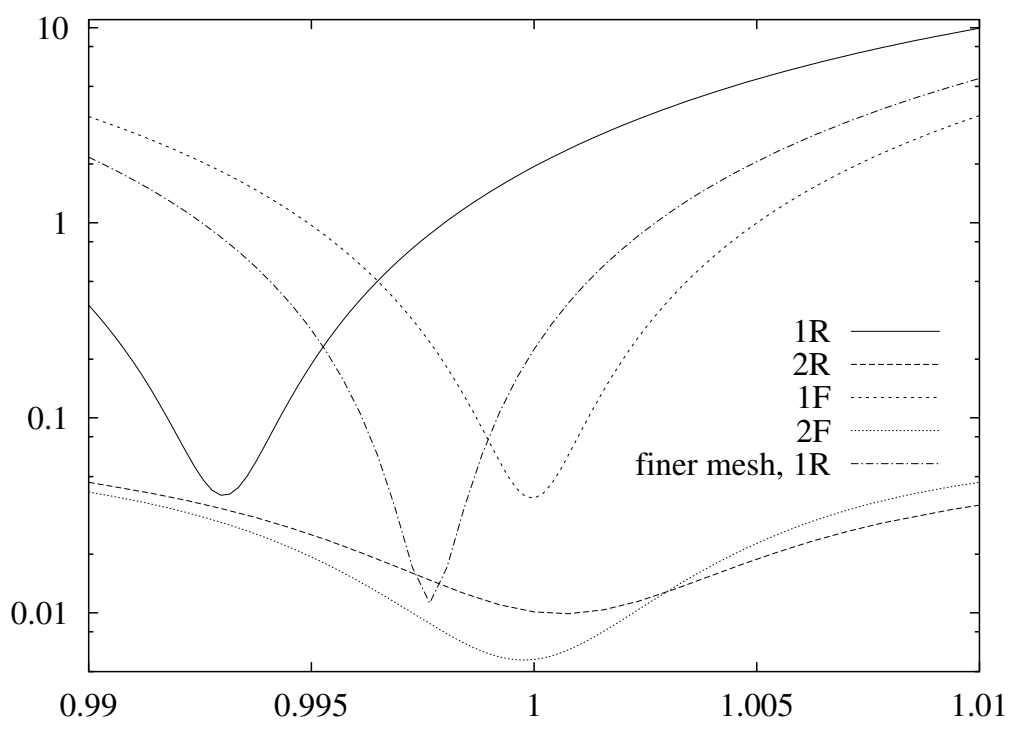

Figure 6: A magnified plot of $B_{F}^{*}$ as a function of the interrogating frequency $f$ for different coating layers.

We have depicted scattered electric fields $E^{(s)}$ with the angle of incidence $15^{\circ}$ for the non coated missile and the missile with the $2 \mathrm{~F}$ coating in Figures 7 and 8, respectively. While the coated missile still produces strong scattering, the backscattering to the radar seems to be minute. This situation is changed when the interrogating frequency is moved further from the design frequency (i.e., $I_{D}=\{1\}$ ) or when the radar is behind the coated missile. The scattered fields for two such cases are shown in Figure 9 for interrogating frequency $f=0.6 \mathrm{GHz}$. and in Figure 10 with the incident angle $165^{\circ}$. To further understand the scattering calculations, we have plotted the radar cross sections for the missiles with the coating layers made of the materials $1 \mathrm{R}, 2 \mathrm{R}, 1 \mathrm{~F}$, and $2 \mathrm{~F}$ as well as for the non coated missile in Figures 11 and 12 . We remark that in the design we used $B_{F}$ which is based on the incident angles between $0^{\circ}$ and $30^{\circ}$ (i.e., (8) with $A=[0,30]$ and $Q$ the uniform distribution on this support region) while the RCS captures reflections in all directions. The plots show very clearly that the material $1 \mathrm{~F}$ reduces the RCS in the sector of interest tens of decibels more effectively than the material $1 \mathrm{R}$. As we already concluded earlier, this is probably due to some extent to the finite element discretization error. 


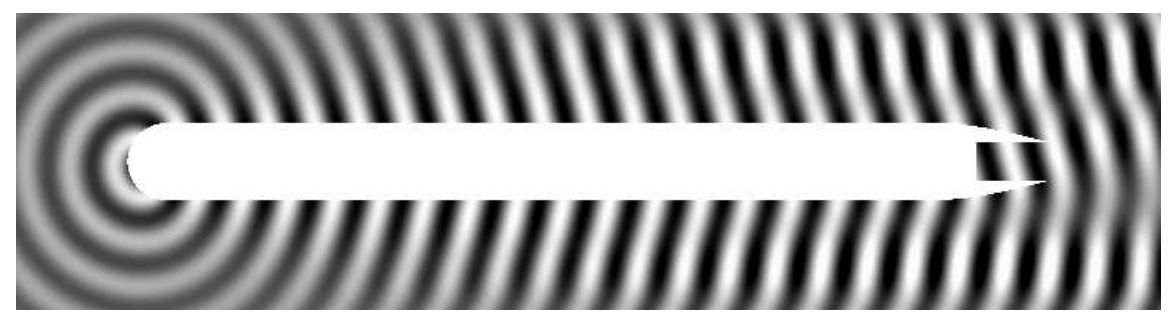

Figure 7: The backscattering without coating layer when the interrogating frequency is $f=1.0$ $\mathrm{GHz}$ and the incident angle is $\alpha=15^{\circ}$.

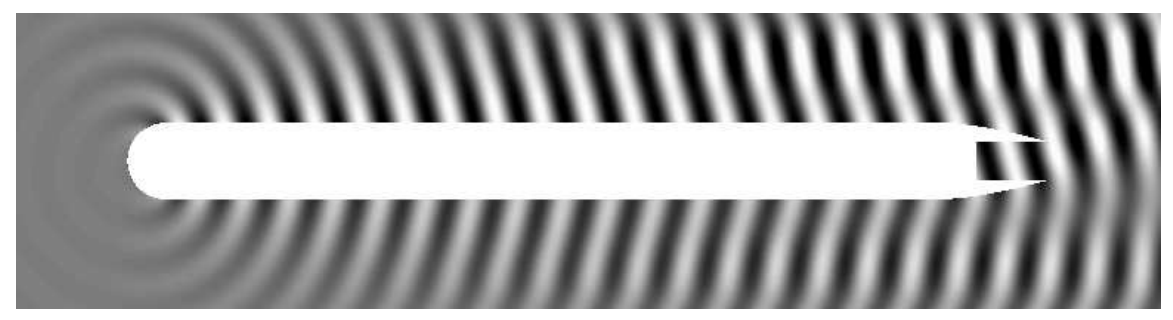

Figure 8: The backscattering with a coating layer made of the material $2 \mathrm{~F}$ when the interrogating frequency is $f=1.0 \mathrm{GHz}$ and the incident angle is $\alpha=15^{\circ}$.

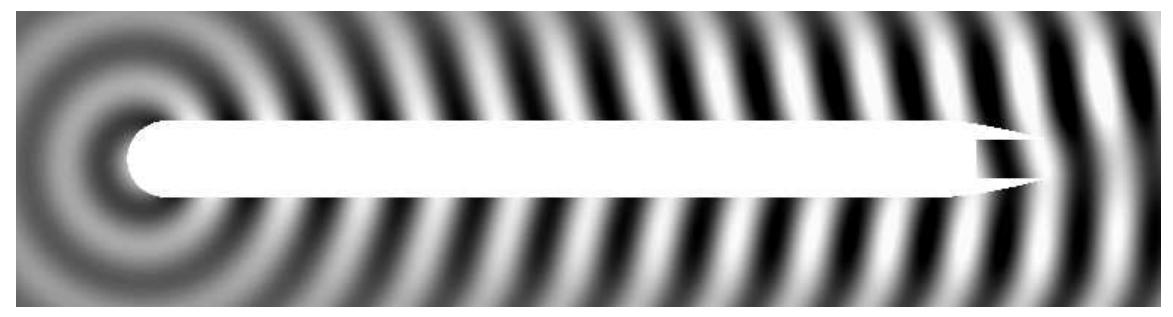

Figure 9: The backscattering with a coating layer made of the material $2 \mathrm{~F}$ when the interrogating frequency is $f=0.6 \mathrm{GHz}$ and the incident angle is $\alpha=15^{\circ}$.

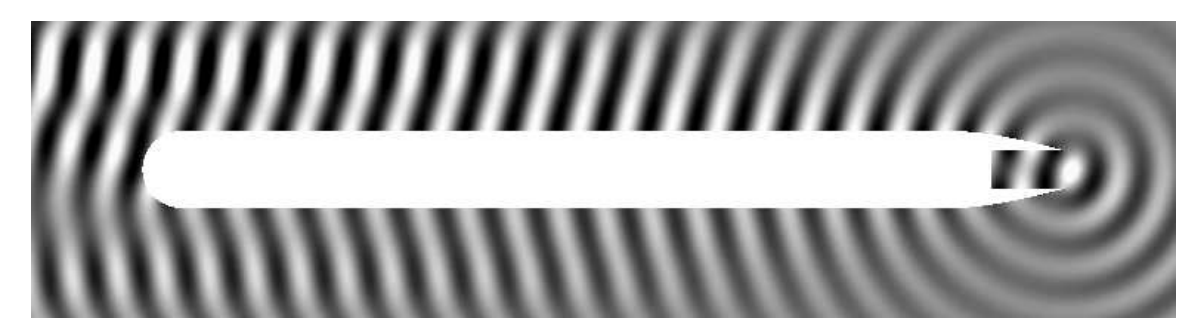

Figure 10: The backscattering with a coating layer made of the material $2 \mathrm{~F}$ when the interrogating frequency is $f=1.0 \mathrm{GHz}$ and the incident angle is $\alpha=165^{\circ}$. 

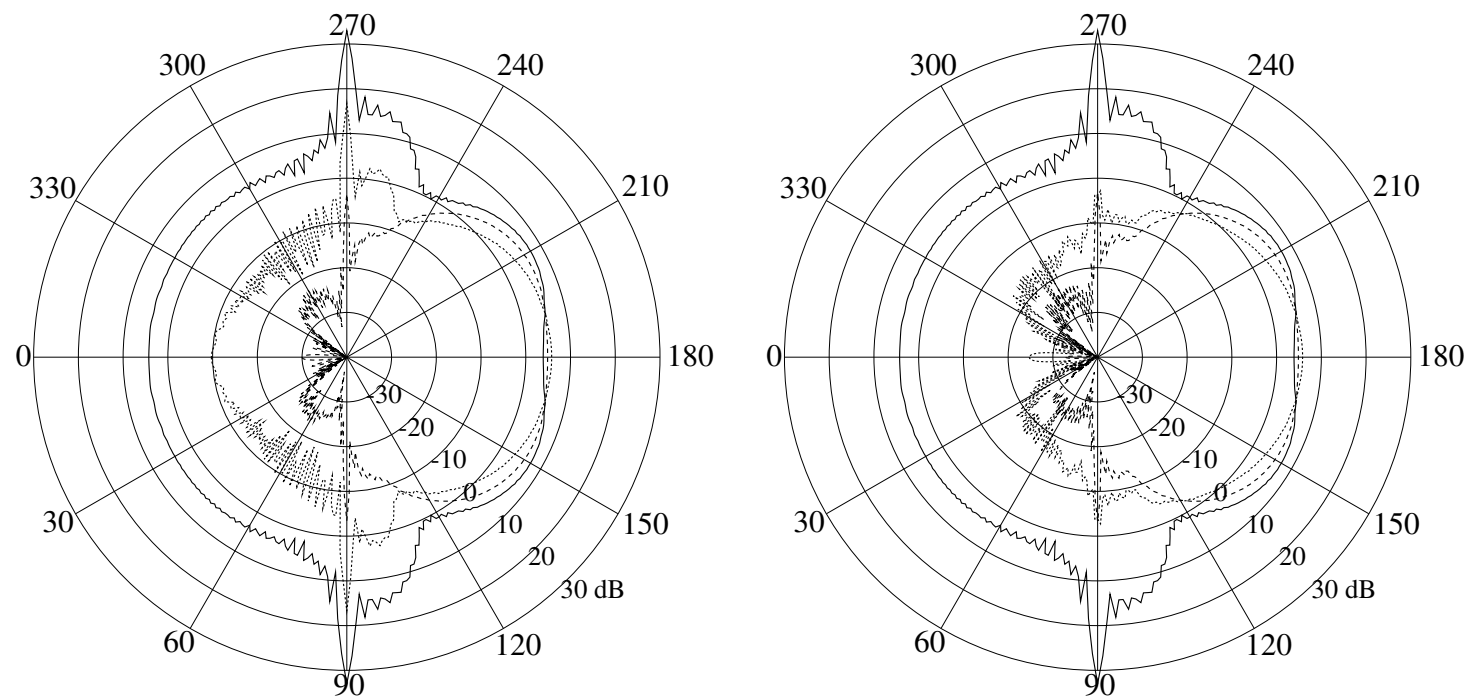

Figure 11: The radar cross sections at $1.0 \mathrm{GHz}$ for no coating, 1R, 2R (left) and no coating, 1F, 2F (right) (lines: solid, dotted, dashed, respectively).
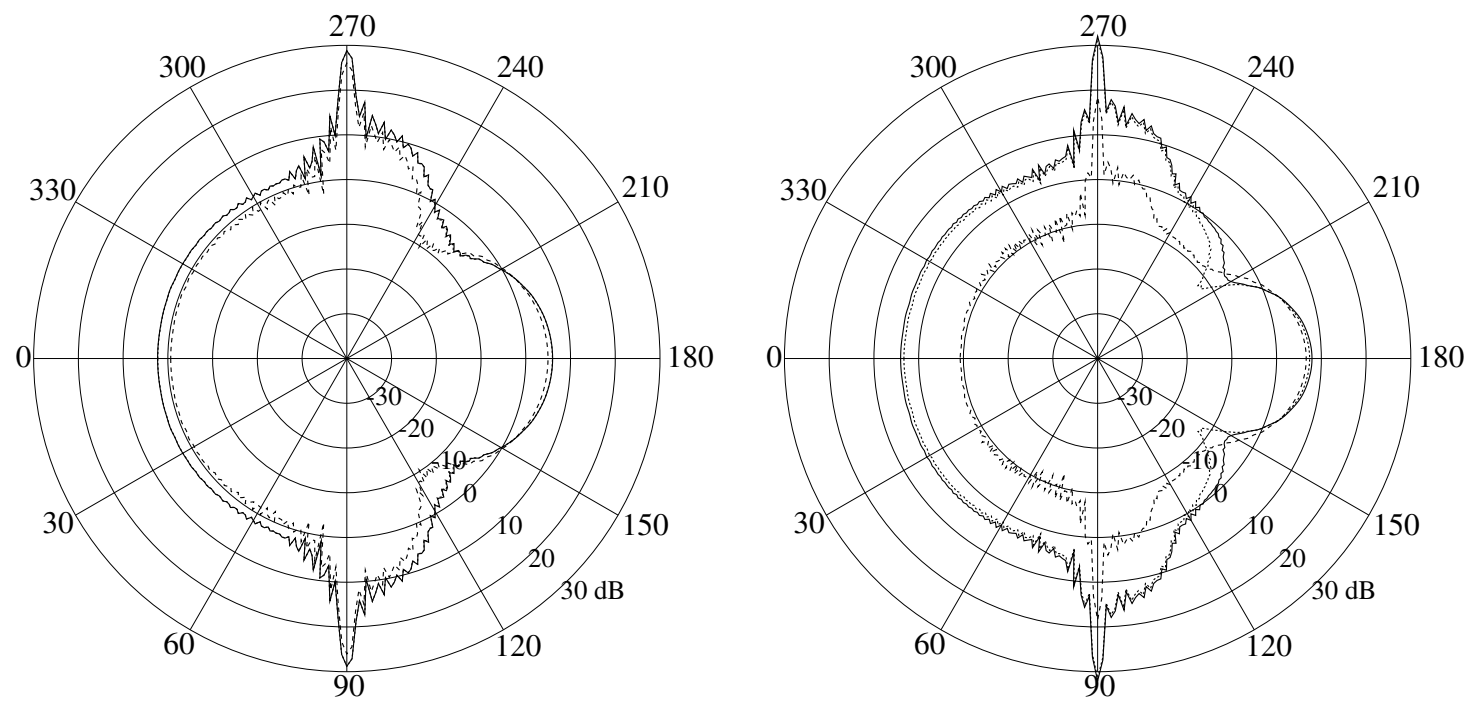

Figure 12: The radar cross sections at $0.6 \mathrm{GHz}$ (left) and $0.9 \mathrm{GHz}$ (right) for no coating, $1 \mathrm{~F}$ and $2 \mathrm{~F}$ materials (lines: solid, dotted and dashed, respectively). 


\subsection{Frequencies around $0.4 \mathrm{GHz}$}

In this section we report on results when we optimize the material parameters for evader's design frequencies $I_{D}$ around $0.4 \mathrm{GHz}$ using the reflection coefficient based $B_{R}$. For lower frequencies like $0.4 \mathrm{GHz}$ one might expect the plane analysis to yield slightly less accurate predictions, since less detail is clearly imaged with decreasing frequency and increasing wavelength. For these optimizations the set $\mathcal{M}$ of feasible material parameters is given by (13) with $\epsilon_{r}^{\max }=$ $10000+2000 i$. The results are reported in Table 2 . We remark that the slightly higher value of $\max B_{R}^{D}$ for the material $5 \mathrm{R}$ than for the material $4 R$ is due to the rounding of the material parameters.

\begin{tabular}{c|c|c|c|c|c|c} 
material & $I_{D}$ & $\mu_{r}^{\max }$ & $\epsilon_{r}^{*}$ & $\mu_{r}^{*}$ & $\max B_{R}^{D}$ & $\max B_{F}^{D}$ \\
\hline \hline no & $\{0.4\}$ & & $1+0 i$ & $1+0 i$ & $1.00 \times 10^{-0}$ & $2.95 \times 10^{+1}$ \\
\hline \hline 4R & $\{0.4\}$ & $1+0 i$ & $5625.4+95.5 i$ & $1+0 i$ & $5.13 \times 10^{-5}$ & $4.03 \times 10^{-0}$ \\
\hline $5 \mathrm{R}$ & $\{0.4\}$ & $10+2 i$ & $558.6+29.3 i$ & $10+1.2 i$ & $8.84 \times 10^{-5}$ & $1.43 \times 10^{-2}$ \\
\hline $6 \mathrm{R}$ & {$[0.32,0.5]$} & $10+2 i$ & $549.3+150.0 i$ & $10+2 i$ & $6.69 \times 10^{-1}$ & $1.65 \times 10^{+1}$
\end{tabular}

Table 2: Optimized coating materials 4R, 5R, 6R using the reflection coefficient $R$ compared to no coating results.

In Figure 13 we graph the values of $B_{R}^{*}(f)=B_{R}\left(\epsilon_{r}^{*}, \mu_{r}^{*}, f\right)$ as a function of the interrogating frequency $f$ for the missiles coated with the materials $4 \mathrm{R}, 5 \mathrm{R}$, and $6 \mathrm{R}$ defined in Table 2 . For the same missiles the values of $B_{F}^{*}$ are plotted in Figure 15. In order to facilitate comparison of $B_{R}^{*}$ and $B_{F}^{*}$ we also again plot the ratio of $B_{F}^{*}$ for the coated missiles to $B_{F}(1,1, f)$ for the non coated missile in Figure 14. Again the modulus of the reflection coefficient $R$ has similar behavior but slightly larger values than the relative value $B_{F}\left(\epsilon_{r}^{*}, \mu_{r}^{*}, f\right) / B_{F}(1,1, f)$. One noticeable difference is the slight shift of the location of the secondary reduction of reflection around $f=1.2 \mathrm{GHz}$. We presume this to be due to the finite element discretization error.

From Figure 14 we see that for the material $4 \mathrm{R}$ the reduction in the backscattering is about 10 decibels for interrogating frequencies within $\pm 1 \%$ of $0.4 \mathrm{GHz}$. For the material $5 \mathrm{R}$ the reduction is about 10 and 20 decibels for interrogating frequencies within $\pm 6 \%$ and $\pm 2 \%$, respectively, of $0.4 \mathrm{GHz}$. The interrogator only needs to change the interrogating frequency about $10 \%$ from $0.4 \mathrm{GHz}$ to render ineffective the coating layer of evader's missile made of the material $4 \mathrm{R}$ or 5R. Thus, we find that the frequency range of high absorption is narrower for the materials optimized for $I_{D}=\{0.4\} \mathrm{GHz}$ than for those optimized with $I_{D}=\{1.0\} \mathrm{GHz}$. Again a dual frequency radar using, for example, interrogating frequencies of 0.4 and $1.4 \mathrm{GHz}$ should be able to detect missiles with any of the optimized coating layers with $I_{D}=\{0.4\} \mathrm{GHz}$ even though one of the operating frequencies was used to design the coating.

Figure 16 depicts the RCS for the non coated missile and the missiles with the coating layer made of the materials $4 \mathrm{R}$ and $5 \mathrm{R}$. The use of the material $5 \mathrm{R}$ obtained by optimizing both permittivity and permeability leads to approximately $30 \mathrm{~dB}$ reduction in the sector of interest $[0,30]^{\circ}$. The use of the permittivity $\epsilon_{r}$ optimized material $4 \mathrm{R}$ leads to only about 10 $\mathrm{dB}$ reduction in the considered sector. The frequency range of high absorption is narrow for the material 4R. Finite element discretization error is probably shifting the location of maximum absorption about 1\% (similar to the behavior of the curve for the material $1 \mathrm{R}$ in Figure 6 ). Thus, the modest reduction in the RCS is probably due to this. 


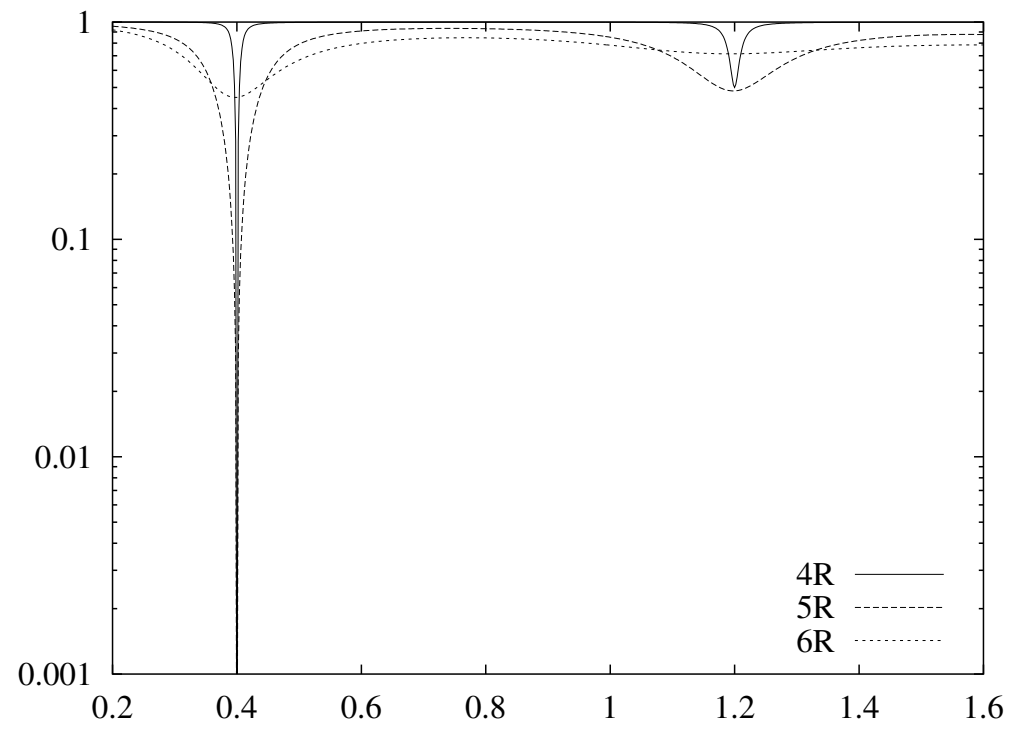

Figure 13: The strength of backscatter $B_{R}^{*}$ as a function of interrogating frequency $f$ for different coating layers.

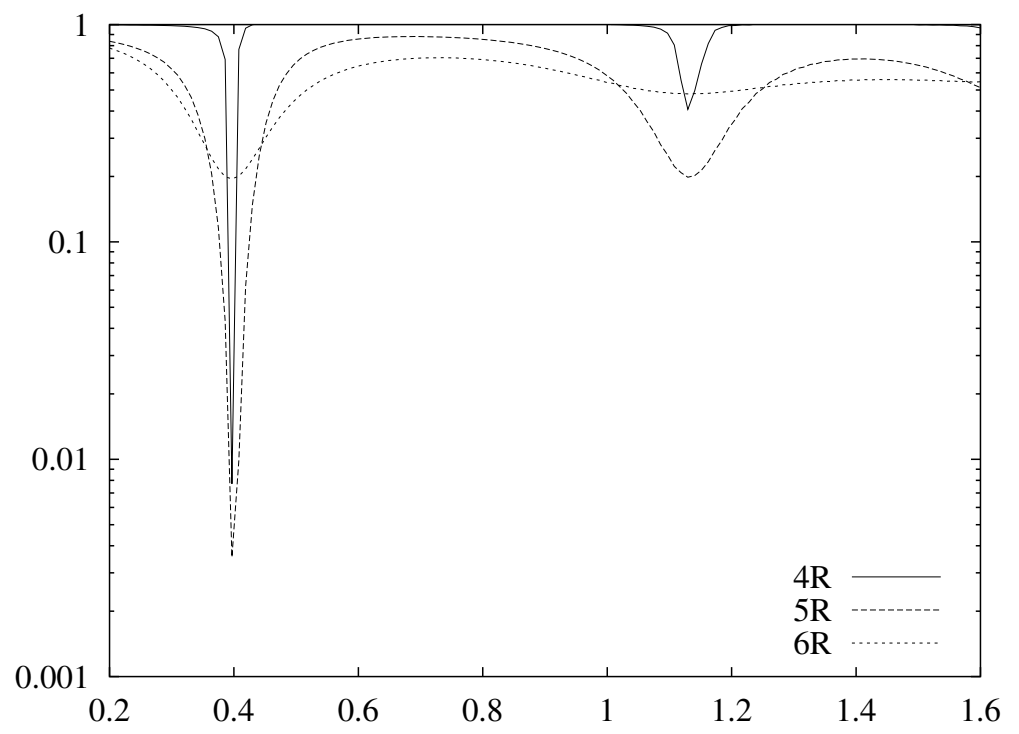

Figure 14: The normalized strength of backscatter $B_{F}\left(\epsilon_{r}^{*}, \mu_{r}^{*}, f\right) / B_{F}(1,1, f)$ as a function of the interrogating frequency $f$ for different coating layers. 


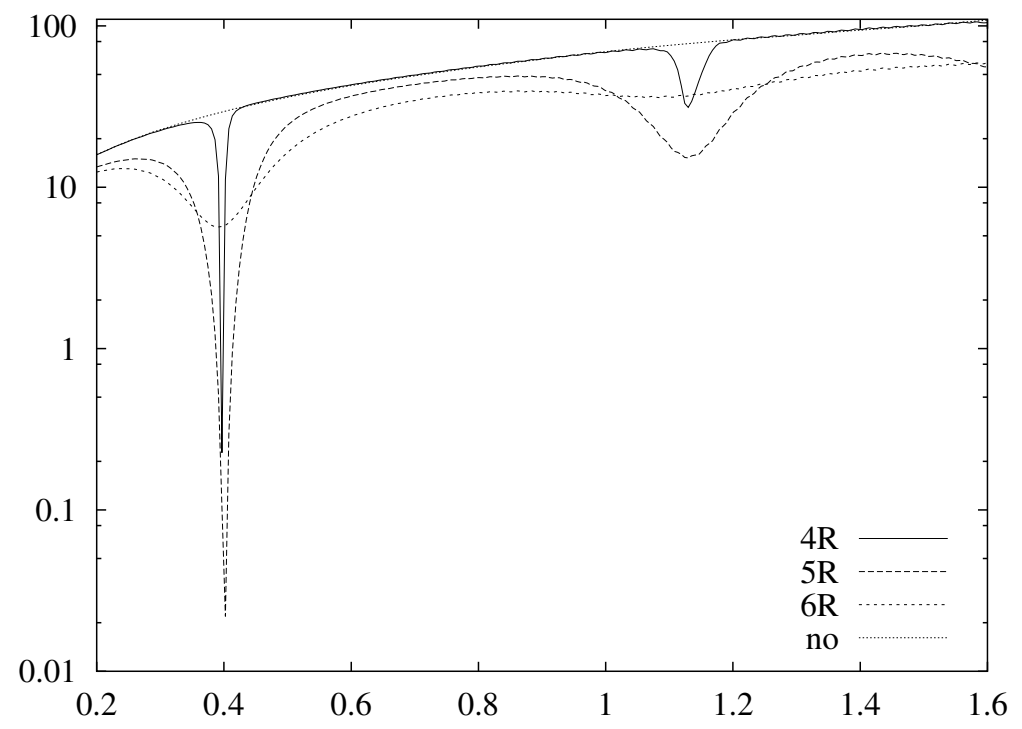

Figure 15: The strength of backscatter $B_{F}^{*}$ as a function of interrogating frequency $f$ for different coating layers.

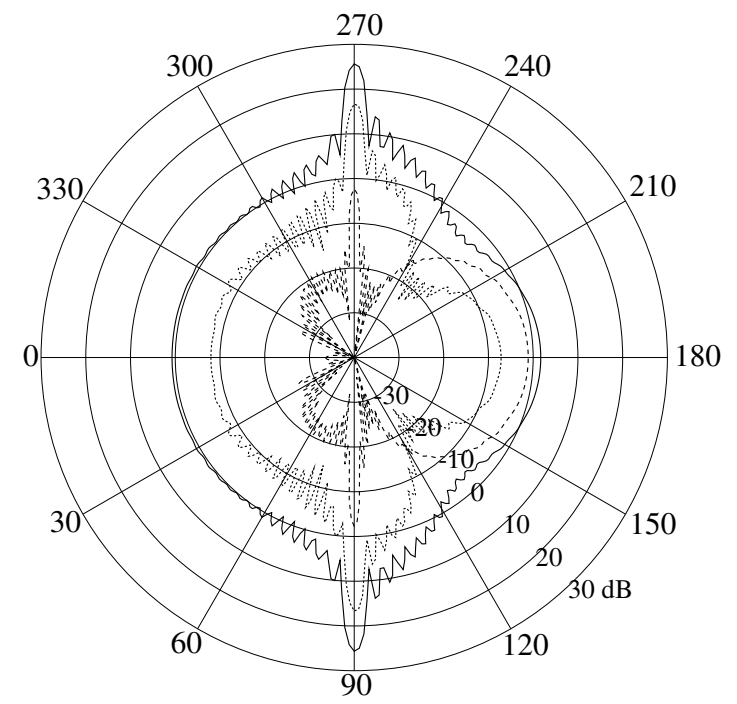

Figure 16: The radar cross sections at $0.4 \mathrm{GHz}$ for no coating, 4R, 5R (line: solid, dotted, dashed, respectively). 


\subsection{Permittivity optimizations using a simplified geometry}

In this section we consider the optimization of the relative permittivity $\epsilon_{r}$ with the fixed relative permeability $\mu_{r}=1$ using a simplified geometry for the cruise missile. More precisely, we replace the cavity modeling the exhaust nozzle by a plane which is also coated. The simplified geometry is shown in Figure 17. The values of $B_{F}$ in the angle range $[0,30]^{\circ}$ for all interrogating frequencies in the range $[0.2,1.6] \mathrm{GHz}$ differ from those for the original geometry in Figure 1 and those for the simplified one in Figure 17 without a coating layer by less than $0.2 \%$. Thus, the curves corresponding to the non coated missiles in Figures 5 and 19 below are essentially the same. We conclude that at least with a non coated missile it is not essential to model the exhaust nozzle when the incident angles are in the range $[0,30]^{\circ}$.

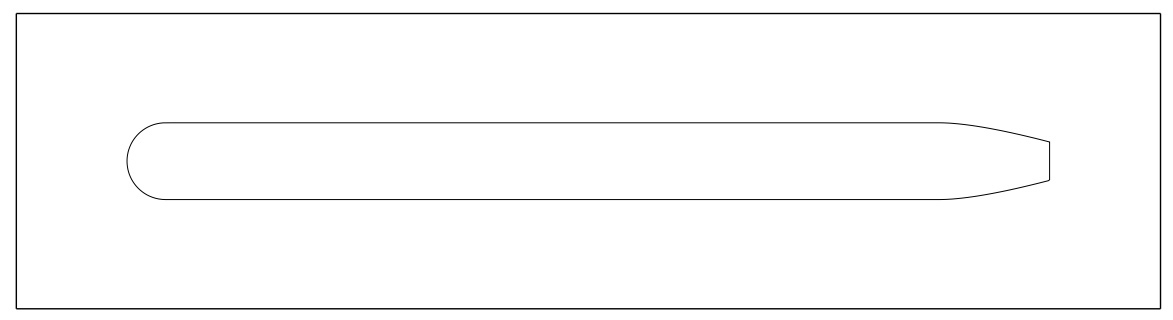

Figure 17: The simplified geometry modeling the cruise missile and the corresponding computational domain.

In the following we choose evader's design frequencies $I_{D}$ to be either $\{0.8\},\{1.2\}$, or $\{1.6\}$ $\mathrm{GHz}$ and the evader performs the optimization using the reflection coefficient based $B_{R}$ resulting in values of $\epsilon_{r}^{*}=1407+48 i, 625+32 i$, or $352+24 i$, respectively. These optimal relative permittivities at the design frequencies are given in Table 3 along with the values of $B_{F}\left(\epsilon_{r}, 1, f\right)$ based on the far field pattern $F$ for the interrogating frequencies $f=0.2,0.4, \ldots, 1.6 \mathrm{GHz}$. Figure 19 depicts the values of $B_{F}^{*}$ in this interrogating frequency range from $0.2 \mathrm{GHz}$ to 1.6 GHz. For a comparison we have also plotted the corresponding $B_{R}^{*}$ in Figure 18. Again the reflection coefficient $R$ gives a good prediction for the behavior of $B_{F}^{*}$.

\begin{tabular}{c|r|r|r|r|r|r|r|r}
$\epsilon_{r} \backslash f$ & 0.2 & 0.4 & 0.6 & 0.8 & 1.0 & 1.2 & 1.4 & 1.6 \\
\hline \hline 1 & 15.9 & 29.5 & 42.7 & 56.0 & 68.3 & 82.8 & 94.6 & 108.7 \\
\hline $1407+48 i$ & 15.9 & 29.4 & 41.9 & 2.4 & 67.7 & 83.0 & 94.7 & 108.8 \\
\hline $625+32 i$ & 15.9 & 29.4 & 42.5 & 55.2 & 62.8 & 1.6 & 86.2 & 106.0 \\
\hline $352+24 i$ & 15.9 & 29.5 & 42.6 & 55.7 & 67.1 & 78.6 & 74.4 & 1.4
\end{tabular}

Table 3: The strength of backscatter $B_{F}\left(\epsilon_{r}, 1, f\right)$ based on the far field pattern $F$ for various coating materials and frequencies.

Scattered electromagnetic fields for various frequencies are shown in Figures 20, 21, 23, 24, $26,27,28,30,31$, and 33 when the incident angle $\alpha$ is $15^{\circ}$. The corresponding radar cross sections are shown in Figures 22, 25, 29, 32, 34. For the coated missiles we have also depicted the radar cross sections when the frequency is $0.1 \mathrm{GHz}$ below the design frequency. These plots demonstrate clearly that even a $0.1 \mathrm{GHz}$ change in frequency makes the coating layers ineffective when only the relative permittivity is tuned to maximize the absorption at the design 


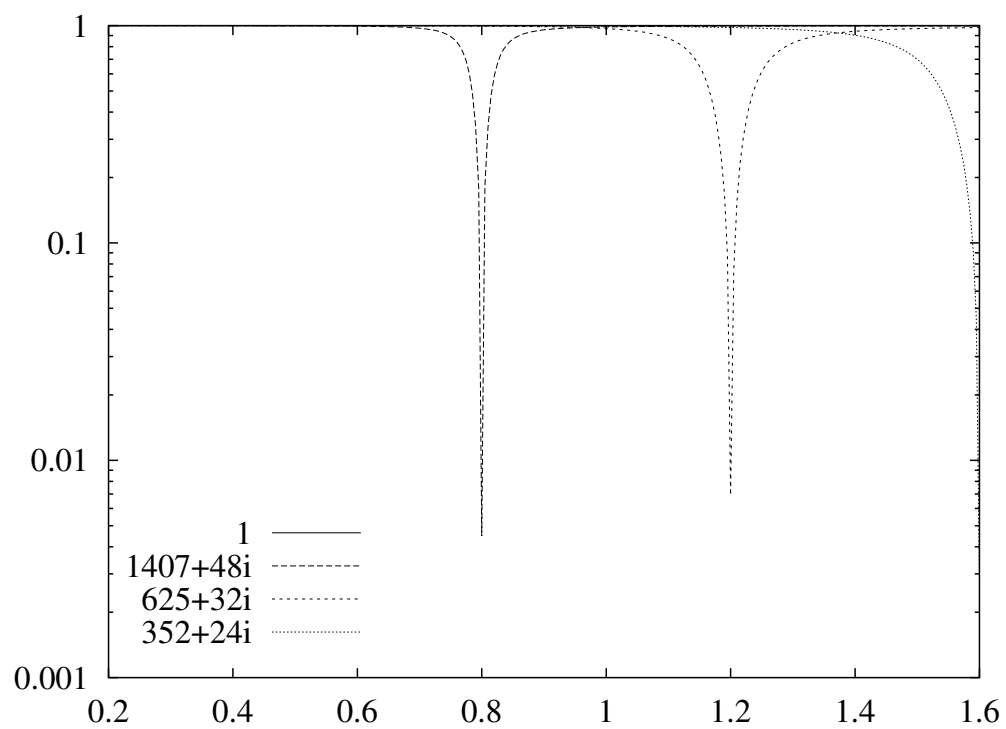

Figure 18: The strength of backscatter $B_{R}^{*}$ as a function of the interrogating frequency $f$ based on the reflection coefficient $R$ for different coating layers.

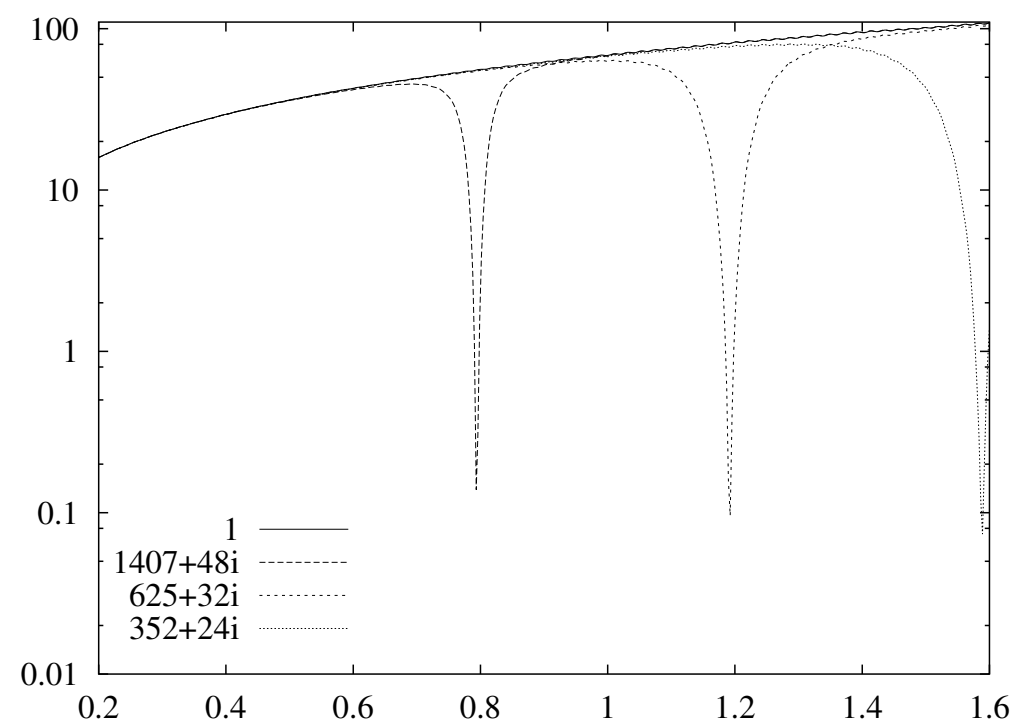

Figure 19: The strength of backscatter $B_{F}^{*}$ as a function of the interrogating frequency $f$ based on the far field pattern $F$ for different coating layers.

frequencies. Thus, one can conclude that for the coating layers considered here, a practical solution for the interrogator is to employ an interrogating frequency about $10 \%$ from the design frequency for the evader coating. An alternative approach for the interrogator is to use a dual frequency radar. 


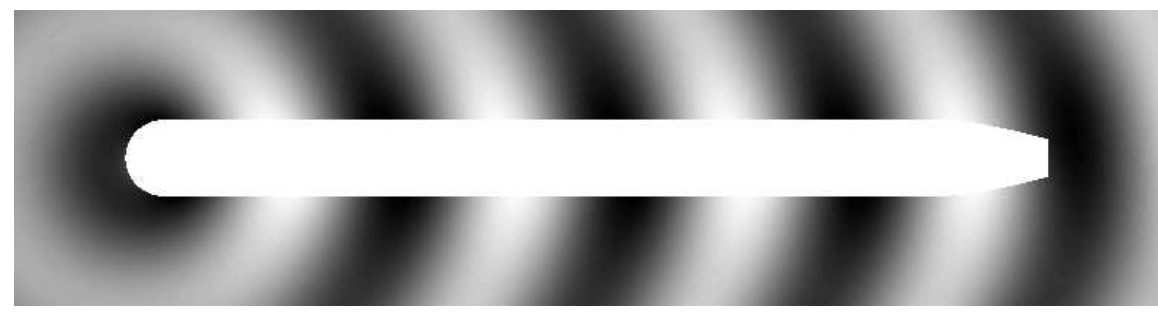

Figure 20: No coating, interrogating frequency $f=0.2 \mathrm{GHz}$, and $\alpha=15^{\circ}$.

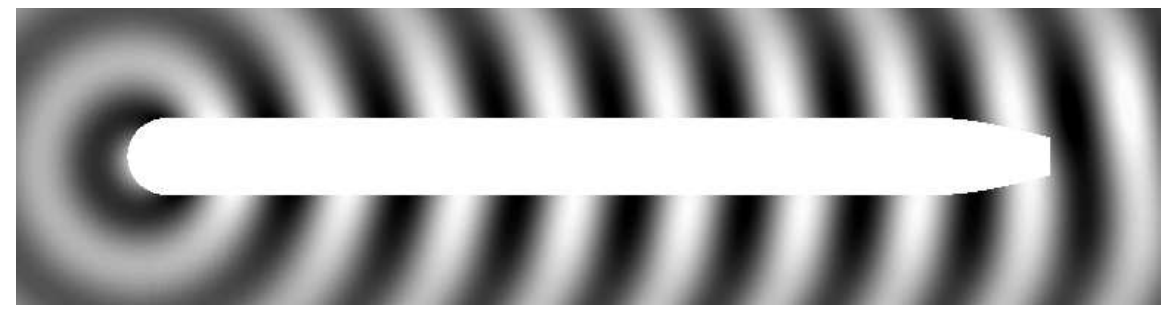

Figure 21: No coating, interrogating frequency $f=0.4 \mathrm{GHz}$, and $\alpha=15^{\circ}$.
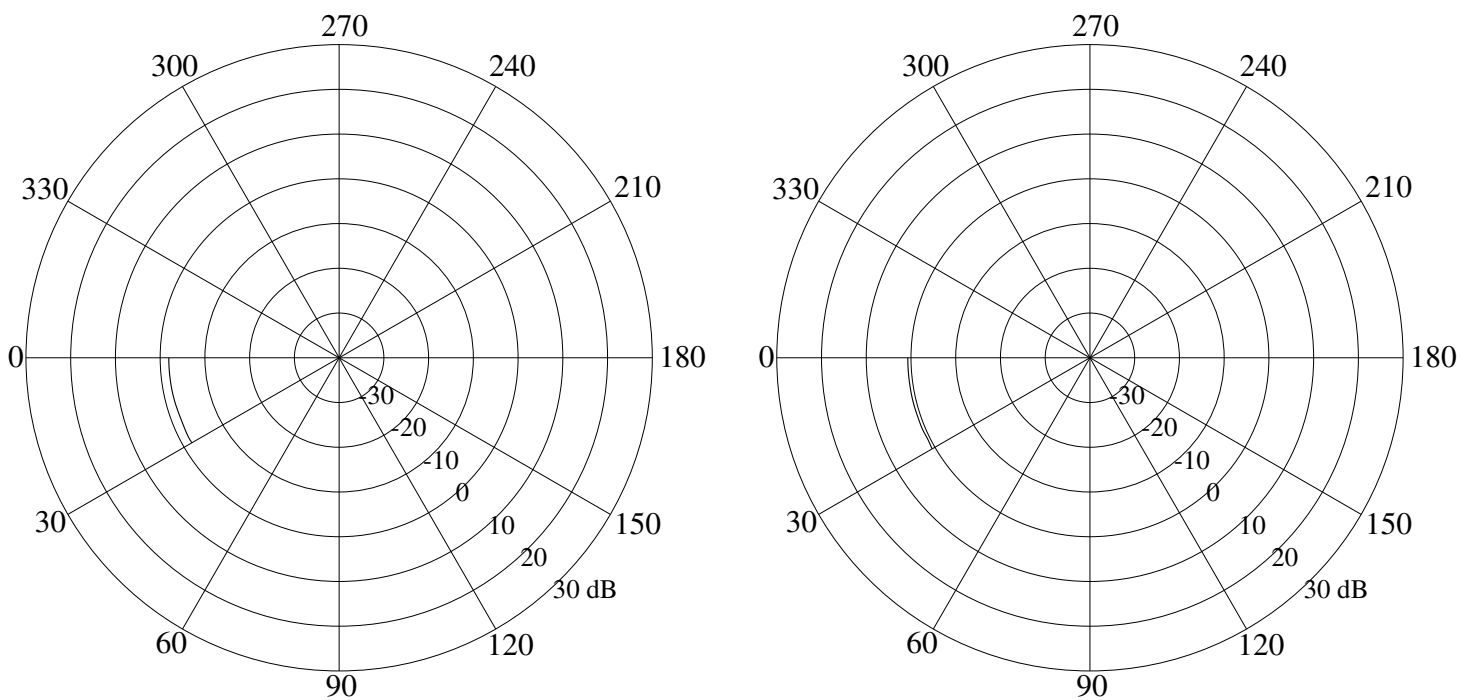

Figure 22: RCS at interrogating frequencies $f=0.2 \mathrm{GHz}$ (left) and $f=0.4 \mathrm{GHz}$ (right) for no coating. 


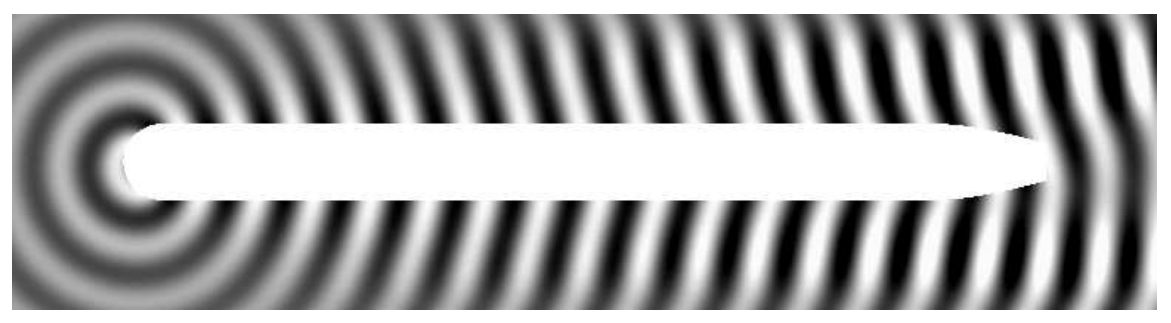

Figure 23: No coating, interrogating frequency $f=0.8 \mathrm{GHz}$, and $\alpha=15^{\circ}$.

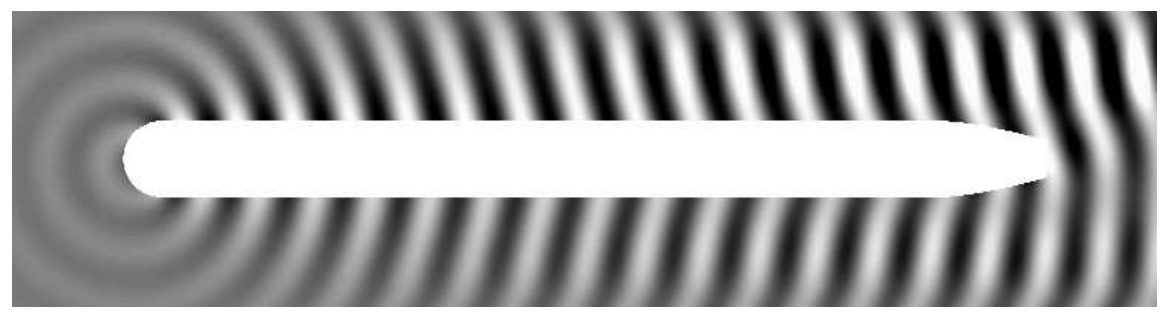

Figure 24: Coating with $\epsilon_{r}^{*}=1407+48 i$, interrogating frequency $f=0.8 \mathrm{GHz}$ and $\alpha=15^{\circ}$.
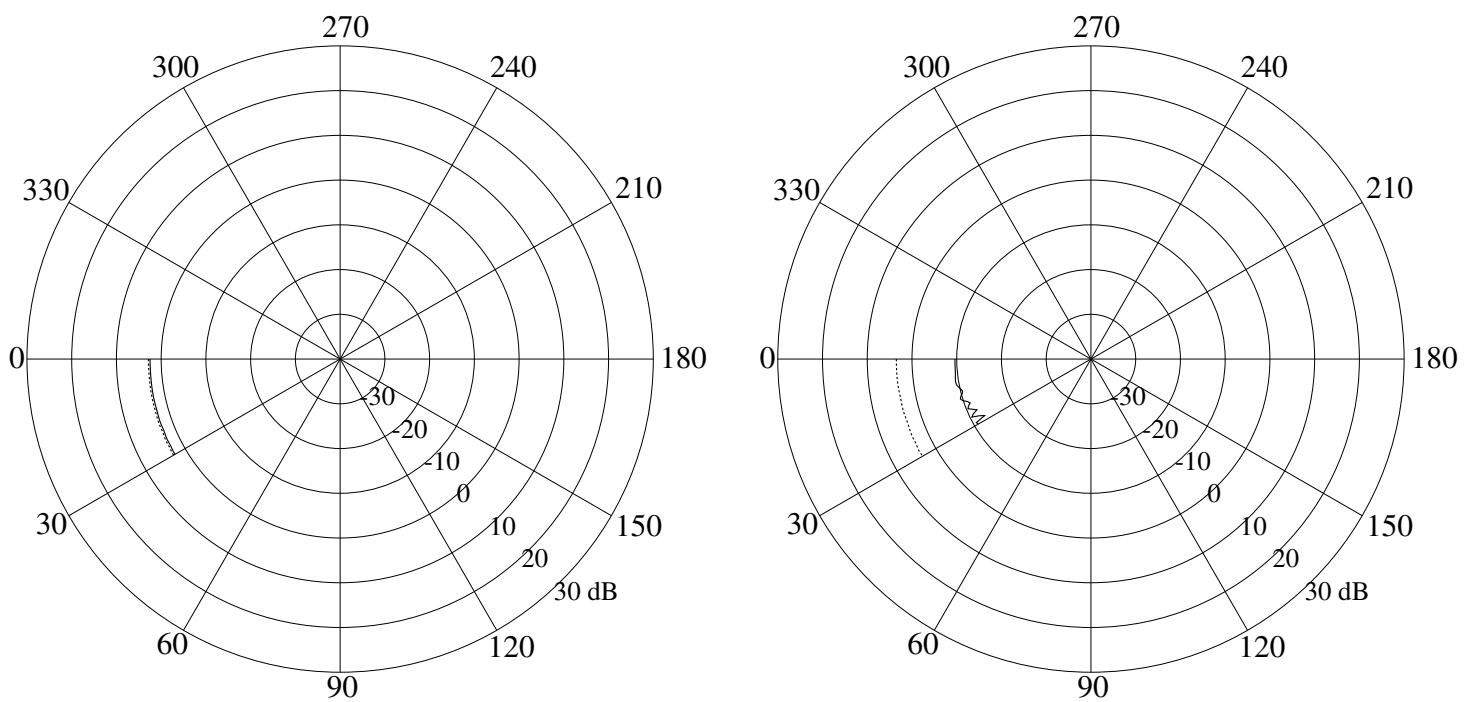

Figure 25: RCS at interrogating frequencies $f=0.7 \mathrm{GHz}$ (left) and $f=0.8 \mathrm{GHz}$ (right) for coating with $\epsilon_{r}^{*}=1407+48 i$ (solid line) and no coating (dotted line). 


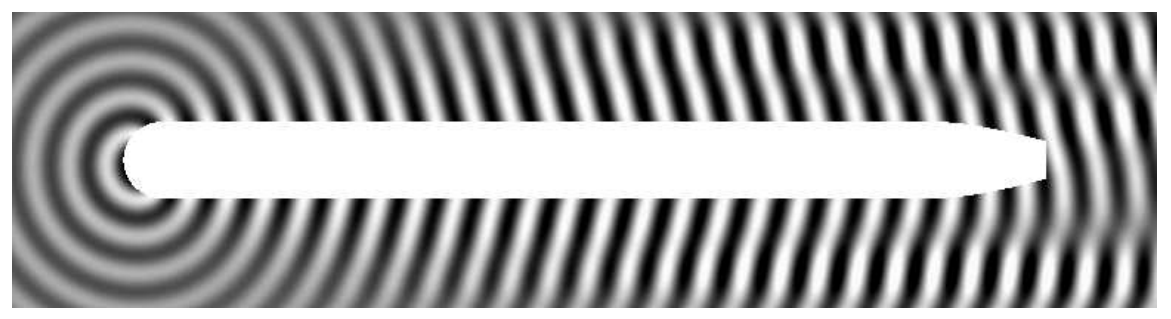

Figure 26: No coating, interrogating frequency $f=1.2 \mathrm{GHz}$, and $\alpha=15^{\circ}$.

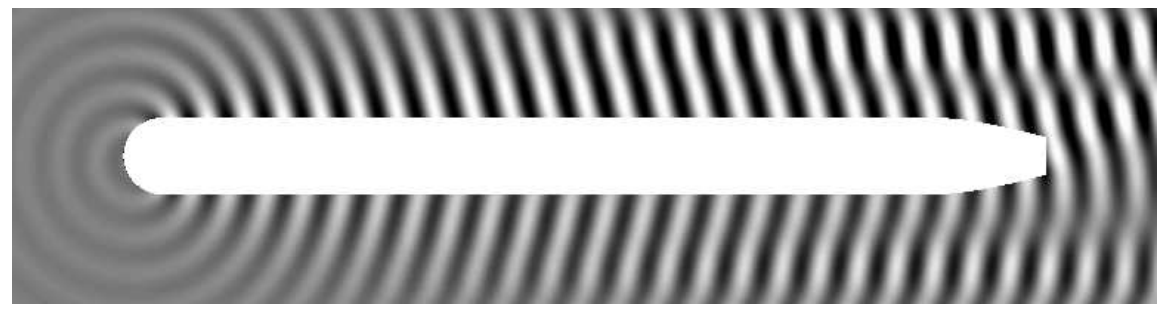

Figure 27: Coating with $\epsilon_{r}^{*}=625+32 i$, interrogating frequency $f=1.2 \mathrm{GHz}$ and $\alpha=15^{\circ}$.

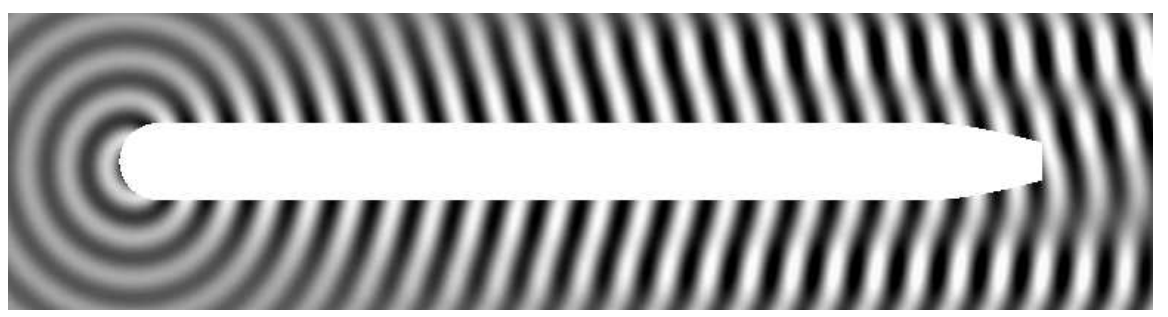

Figure 28: Coating with $\epsilon_{r}^{*}=625+32 i$, interrogating frequency $f=1.1 \mathrm{GHz}$ and $\alpha=15^{\circ}$. 

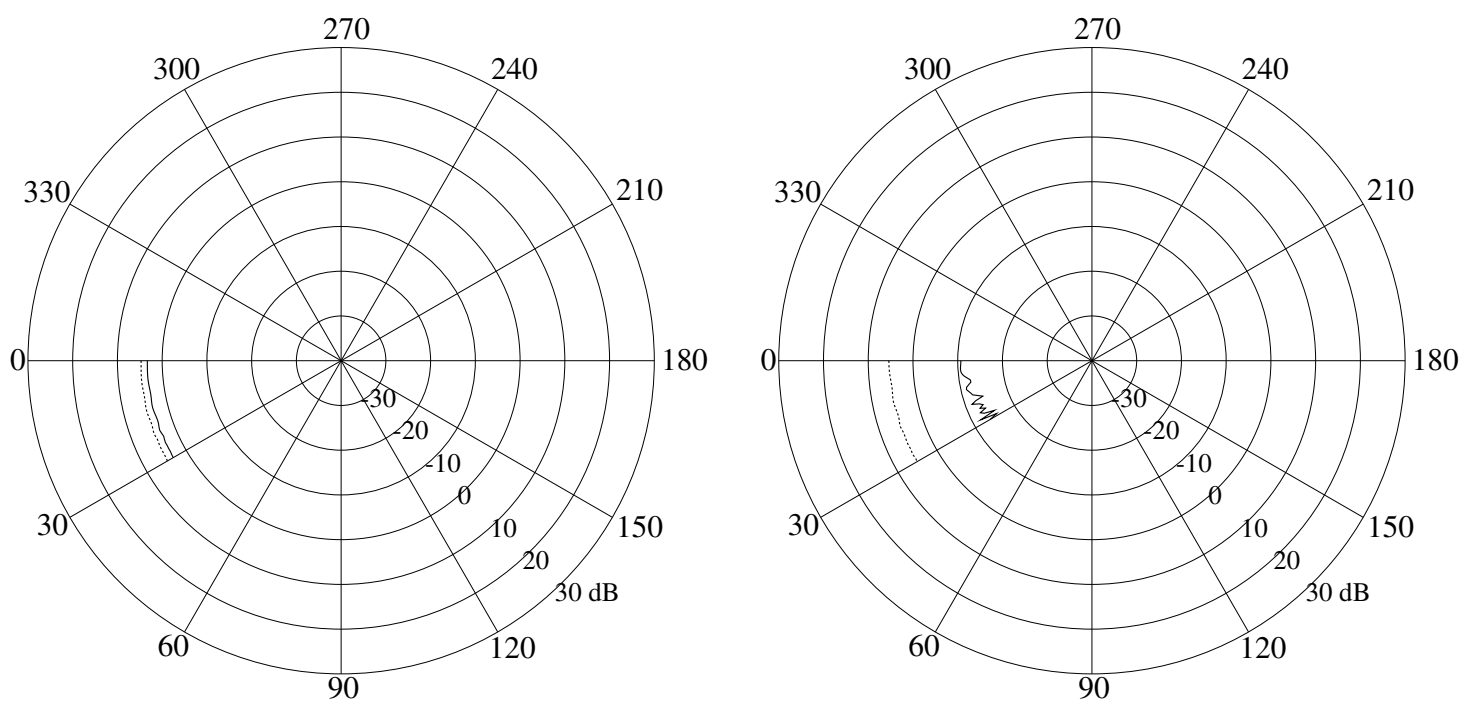

Figure 29: RCS at interrogating frequencies $f=1.1 \mathrm{GHz}$ (left) and $f=1.2 \mathrm{GHz}$ (right) for coating with $\epsilon_{r}^{*}=625+32 i$ (solid line) and no coating (dotted line).

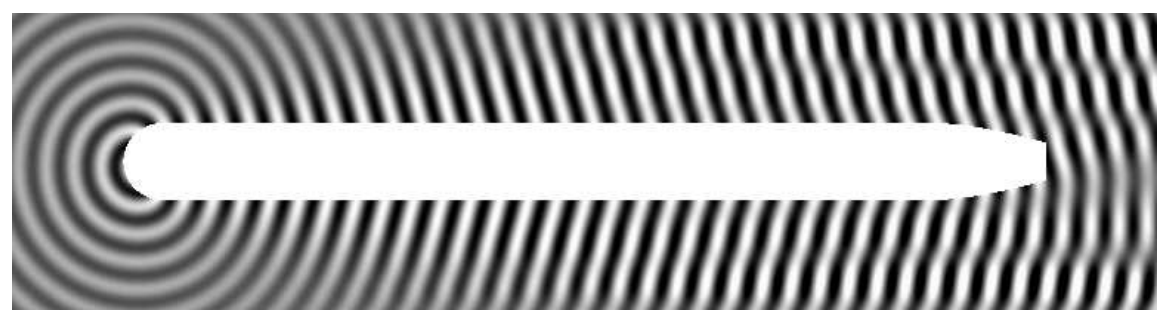

Figure 30: No coating, interrogating frequency $f=1.6 \mathrm{GHz}$, and $\alpha=15^{\circ}$.

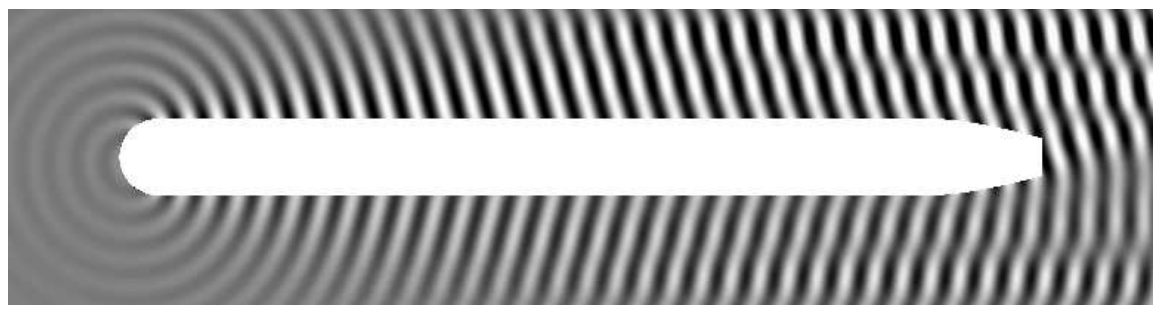

Figure 31: Coating with $\epsilon_{r}^{*}=352+24 i$, interrogating frequency $f=1.6 \mathrm{GHz}$ and $\alpha=15^{\circ}$. 

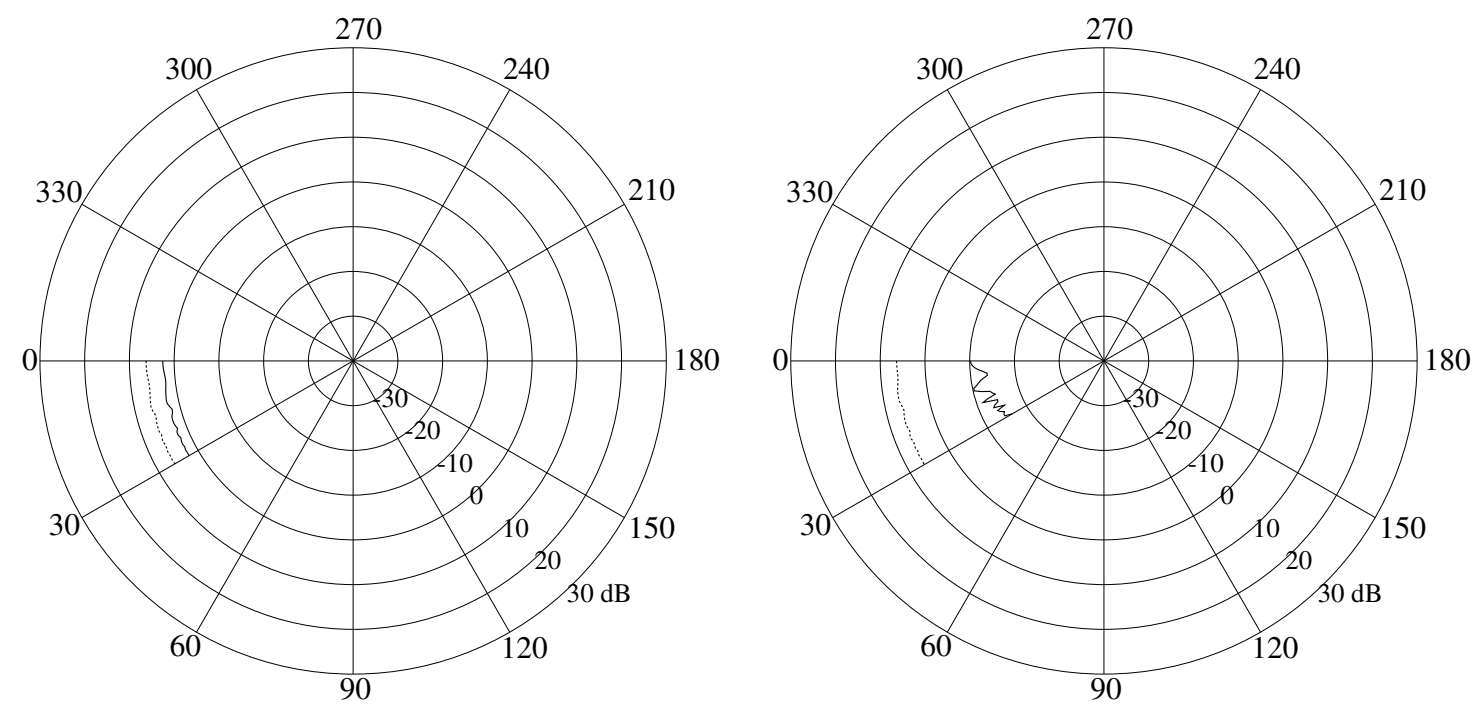

Figure 32: RCS at interrogating frequencies $f=1.5 \mathrm{GHz}$ (left) and $f=1.6 \mathrm{GHz}$ (right) for coating with $\epsilon_{r}^{*}=352+24 i$ (solid line) and no coating (dotted line).

From Figure 19 it can be seen that $B_{F}^{*}(f)$ attains its minimum value about from $0.5 \%$ to $1 \%$ below the design frequency. This is again probably due to the finite element discretization error. As the last experiment we minimized $B_{F}$ instead of $B_{R}$ with respect to the relative permittivity $\epsilon_{r}$ for evader's design frequency $I_{D}=\{1.6\} \mathrm{GHz}$. The optimal value is approximately $\epsilon_{r}^{*}=$ $346.759+24.692 i$ and the associated value of $B_{F}^{D}$ is about 0.096. Thus, the strength of the backscattering is over 10 decibels smaller than for $\epsilon_{r}=352+24 i$. In Figure 33 we show the scattered field for the optimized coating layer. The corresponding radar cross sections are plotted in Figure 34.

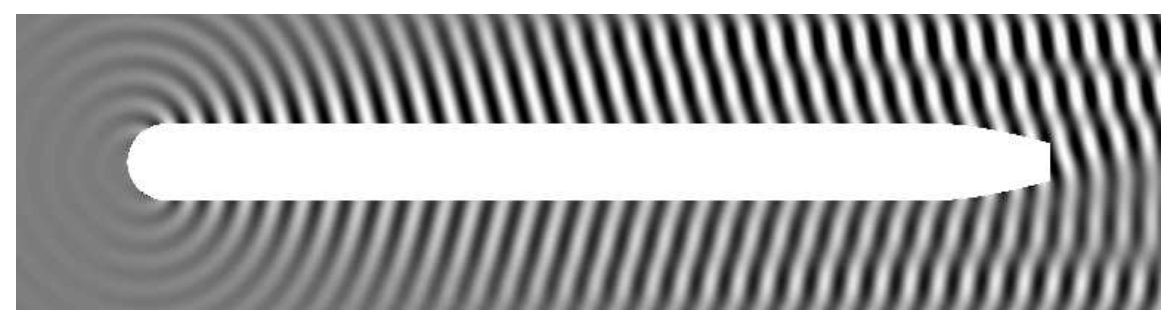

Figure 33: Coating with $\epsilon_{r}^{*}=346.759107+24.6916716 i$, interrogating frequency $f=1.6 \mathrm{GHz}$ and $\alpha=15^{\circ}$. 


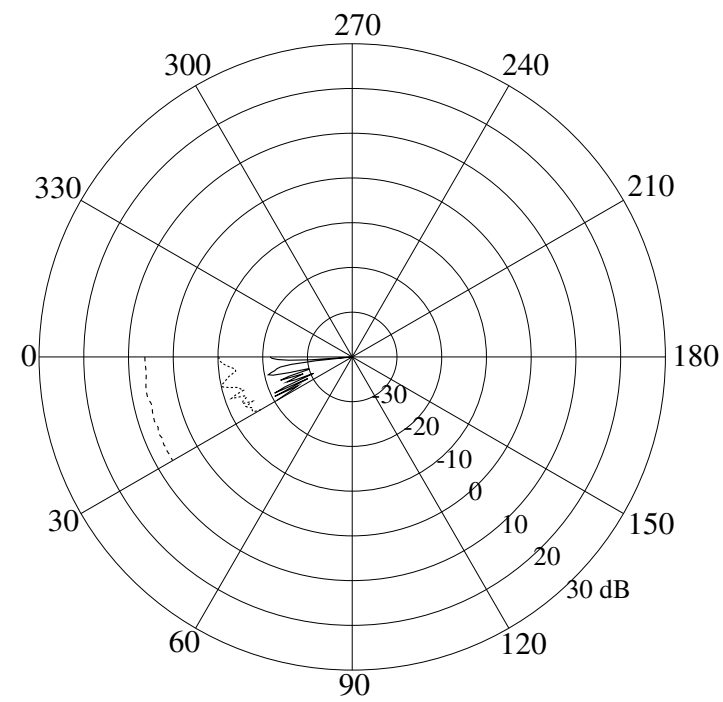

Figure 34: RCS at interrogating frequency $f=1.6 \mathrm{GHz}$ for coating with $\epsilon_{r}^{*}=346.759107+$ $24.6916716 i$ (solid line), $\epsilon_{r}^{*}=352+24 i$ (dotted line), and no coating (dashed line).

\section{Conclusions}

We have considered the electromagnetic backscattering by the crosscut of a perfectly conducting cruise missile which is coated by a homogeneous electromagnetic absorbent material coating of constant thickness. If the evader uses only the relative permittivity $\epsilon_{r}$ of the coating material to reduce the backscattering, then the interrogator can render the coating ineffective by changing the interrogating frequency of the radar by, say, $10 \%$ from evader's design frequency. The evader can make the range of high absorbent frequencies wider by tuning both relative permittivity and magnetic permeability of the coating material. Even with such a coating layer it is not possible to substantially reduce the radar cross section for both high and low frequencies at the same time. Thus, the interrogator should be able to detect the cruise missile by using a dual frequency radar, operating for example at interrogating frequencies $f=0.4$ and $f=1.4 \mathrm{GHz}$ for the coatings in our calculations. For more complex coating layers, say, employing nonlinear materials and multilayering, this conclusion might not be valid.

We also make a useful observation that the reflection coefficient based on a planar geometry gives a fairly accurate and easily computable upper limit for the backscattering for the considered scattering problems. Hence, the interrogator can use the reflection coefficient to quickly and easily determine effective radar operating frequencies once there is sufficient information on the evader's coating layer. The finite element model provides a more reliable but also computationally much more expensive way to analyze the backscattering. Due to the discretization error our finite element model with more than a quarter million unknowns appears to lead to up to $1 \%$ error in the frequency minimizing the backscattering. We were able to reduce this error by refining the discretization, but then the computational cost with the refined model is already too high for optimizations in a modern PC. 
In further studies one could consider more complicated coating layers and three-dimensional geometries. For example, the coating material could be nonlinear leading to the permittivity and permeability properties being frequency dependent. The cruise missile we investigated is a body of revolution (BOR) and, thus, for example, the method proposed in [16] could be used to compute the three-dimensional electromagnetic backscattering. Including fins in the model would lead to a general three-dimensional problem which would require a computational methodology such as the finite elements methods considered in [24].

\section{Acknowledgments}

This research was supported in part by the U.S. Air Force Offices of Scientific Research under grant AFOSR FA9550-04-1-0220. The authors would like to thank Dr. Richard Albanese of the AFRL, Brooks AFB, San Antonio TX, for numerous stimulating and valuable comments and suggestions on this work.

\section{References}

[1] A. Bamberger, P. Joly, And J. E. Roberts, Second-order absorbing boundary conditions for the wave equation: a solution for the corner problem, SIAM J. Numer. Anal., 27 (1990), pp. 323-352.

[2] H. T. Banks AND K. L. BiharI, Modeling and estimating uncertainty in parameter estimation, Inverse Problems, 17 (2001), pp. 95-111.

[3] H. T. Banks, D. Bortz, G. A. Pinter and L. K. Potter, Modeling and imaging techniques with potential for application in bioterrorism, Chapter 6 in Bioterrorism: Mathematical Modeling Applications in Homeland Security, H.T. Banks and C. Castillo-Chavez, eds., Frontiers in Applied Mathematics, SIAM, Philadelphia, 2003, pp. 129-154.

[4] H. T. Banks And N. L. Gibson, Well-posedness of solutions with a distribution of dielectric parameters, Applied Mathematics Letters, 18 (2005), pp. 423-430.

[5] H. T. Banks And N. L. GiBson, Electromagnetic inverse problems involving distributions of dielectric mechanisms and parameters,Tech. Report CRSC-TR05-29, Center for Research in Scientific Computation, North Carolina State University, Raleigh, NC, August, 2005; Inverse Problems, submitted.

[6] H. T. Banks And K. Kunisch, Estimation Techniques for Distributed Parameter Systems, Birkhauser, Boston, 1989.

[7] H. T. Banks And G. A. Pinter, A probabilistic multiscale approach to hysteresis in shear wave propagation in biotissue, SIAM J. Multiscale Modeling and Simulation, 3 (2005), pp. 395-412

[8] H. T. Banks, K. Ito, G. M. Kepler, and J. A. Toivanen, Material surface design to counter electromagnetic interrogation of targets, Tech. Report CRSC-TR04-37, Center for Research in Scientific Computation, North Carolina State University, Raleigh, NC, November, 2004; SIAM J. Appl. Math., submitted. 
[9] F. J. Baron and O. Pironneau, Multidisciplinary optimal design of a wing profile, in Structural Optimization 93, The World Congress on Optimal Design of Structural Systems, J. Herskovits, ed., vol. II, 1993, pp. 61-68.

[10] T. Basar And G. J. Olsder, Dynamic Noncooperative Game Theory, SIAM Publ., Philadelphia, PA, $2^{\text {nd }}$ edition, 1999.

[11] A. Bondeson, Y. Yang, And P. Weinerfelt, Optimization of radar cross sections by a gradient method, IEEE Trans. Antennas Propagation, 40 (2004), pp. 1260-1263.

[12] - Shape optimization for radar cross sections by a gradient method, Internat. J. Numer. Methods Engrg., 61 (2004), pp. 687-715.

[13] M. BRaIn, How cruise missiles work, http://science.howstuffworks.com/cruisemissile2.htm, 2005.

[14] D. Colton And R. Kress, Inverse Acoustic and Electromagnetic Scattering Theory, vol. 93 of Applied Mathematical Sciences, Springer-Verlag, Berlin, $2^{\text {nd }}$ ed., 1998.

[15] P. E. Gill, W. Murray, and M. H. Wright, Practical Optimization, Academic Press Inc., London, 1981.

[16] A. D. Greenwood And J. M. Jin, A noval efficient algorithm for scattering from a complex BOR using vector FEM and cylindrical PML, IEEE Trans. Antennas Propagation, 47 (1999), pp. 620-629.

[17] T. Halleröd, D. Ericsson, And A. Bondeson, Shape and material optimization using gradient methods and the adjoint problem in time and frequency domain, COMPEL, 24 (2005), pp. 882-892.

[18] E. Heikkola, Y. A. Kuznetsov, P. Neittaanmäki, and J. Toivanen, Fictitious domain methods for the numerical solution of two-dimensional scattering problems, J. Comput. Phys., 145 (1998), pp. 89-109.

[19] E. Heikkola, T. Rossi, And J. Toivanen, A domain decomposition technique for two-dimensional scattering problems with coated obstacles, in Innovative Tools for Scientific Computation in Aeronautical Engineering, J. Périaux, P. Joly, O. Pironneau, and E. Oñate, eds., CIMNE, 2001, pp. 183-194.

$[20] \_$- Fast direct solution of the Helmholtz equation with a perfectly matched layer or an absorbing boundary condition, Internat. J. Numer. Methods Engrg., 57 (2003), pp. 20072025 .

[21] J. D. Jackson, Classical Electrodynamics, Wiley \& Sons, New York, 1975.

[22] R. A. E. MÄKinen, J. PÉRIAux., And J. Toivanen, Multidisciplinary shape optimization in aerodynamics and electromagnetics using genetic algorithms, Int. J. Numer. Meth. Fluids, 30 (1999), pp. 149-159. 
[23] R. A. E. MÄkinen And J. Tolvanen, Optimal shape design for Helmholtz/potential flow problem using fictitious domain method, in Proceedings of the 5th AIAA/USAF/NASA/ISSMO Symposium on Multidisciplinary Analysis and Optimization, vol. II, 1994, pp. 529-536.

[24] P. Monk, Finite Element Methods for Maxwell's Equations, Numerical Mathematics and Scientific Computation, Oxford University Press, New York, 2003. 